Article

\title{
New Members of the Cinchona Alkaloids Family: Assembly of the Triazole Heterocycle at the $6^{\prime}$ Position
}

\author{
Catalin Vasile Maftei ${ }^{1}$, Martin Heiko Franz ${ }^{1}$, Christian Kleeberg ${ }^{2}$ and Ion Neda ${ }^{2, *}$ \\ 1 InnoChemTech GmbH, Hagenring 30, 38106 Braunschweig, Germany; catalinv.maftei@gmail.com (C.V.M.); \\ franz@innochemtech.de (M.H.F.) \\ 2 Institut für Anorganische und Analytische Chemie, Technische Universität 'Carola-Wilhelmina' \\ Braunschweig, Hagenring 30, 38106 Braunschweig, Germany; ch.kleeberg@tu-braunschweig.de \\ * Correspondence: i.neda@tu-braunschweig.de
}

check for updates

Citation: Maftei, C.V.; Franz, M.H.; Kleeberg, C.; Neda, I. New Members of the Cinchona Alkaloids Family: Assembly of the Triazole Heterocycle at the $6^{\prime}$ Position. Molecules 2021, 26, 3357. https://doi.org/10.3390/ molecules26113357

Academic Editor: Ionel Mangalagiu

Received: 30 April 2021

Accepted: 28 May 2021

Published: 2 June 2021

Publisher's Note: MDPI stays neutral with regard to jurisdictional claims in published maps and institutional affiliations.

Copyright: (c) 2021 by the authors. Licensee MDPI, Basel, Switzerland. This article is an open access article distributed under the terms and conditions of the Creative Commons Attribution (CC BY) license (https:/ / creativecommons.org/licenses/by/ $4.0 /)$.

\begin{abstract}
The substance class of the well-known Cinchona alkaloids is widened by $6^{\prime}$-Aminocinchonine and $6^{\prime}$-Amino-cinchonidine, novel compounds which incorporate a primary amino function in the quinolinic ring system. These key intermediates open the field for a range of fruitful chemistry. Here is described a short and direct pathway for the synthesis of triazole containing derivatives of the above-mentioned substances using the [3+2] Huisgen cycloaddition. For this purpose, the amines were first converted into the corresponding azides. Based on this, non-substituted and silyl-protected triazoles were synthesized as examples. Furthermore, didehydrated derivatives of quincorine and quincoridine were used as addition partners, resulting in compounds that carry the quinuclidine ring of the cinchona alkaloids at both ends. Some of these compounds were examined radiographically to investigate the position of the quinuclidine ring to the triazole. The solid-state structures of compounds 10, 11 and 28 were determined by X-ray diffraction analyses.
\end{abstract}

Keywords: [3 + 2] cycloaddition; Cinchona alkaloids; triazoles; quincorine; quincoridine

\section{Introduction}

To the best of our knowledge, the pseudo-enantiomeric compounds 6 -Amino-cinchonine 1 and $6^{\prime}$-Amino-cinchonidine $\mathbf{2}$ (Figure 1) are not described in the literature. However, there are a few references to the hydrogenated derivatives, the most recent of which dates from 1960 [1]. It is therefore all the more gratifying that these forgotten derivatives of the Cinchona alkaloids are now rationally available.

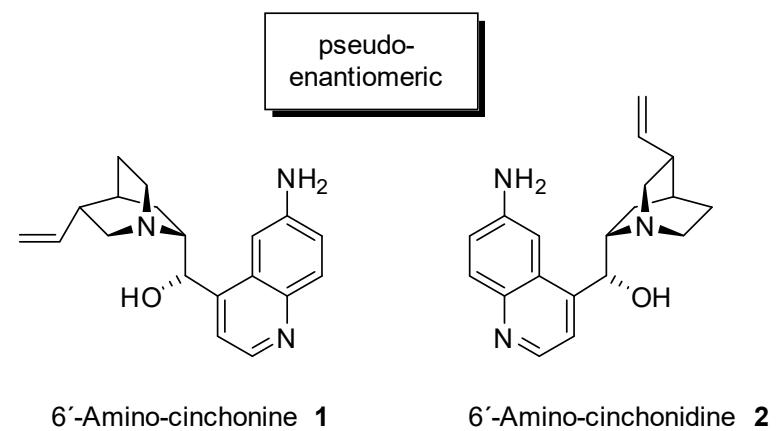

Figure 1. 6'-Amino-cinchonine 1 and $6^{\prime}$-Amino-cinchonidine 2, a pair of pseudo-enantiomeric derivatives of the Cinchona alkaloids quinidine and quinine.

The benefits of Cinchona alkaloids and their derivatives are widely known. Quinine is still used as an anti-malarial in medicine and as a bittering agent in the beverage industry. Cupreins and cupreidines, hydroxy analogues of amines 1 and 2, are currently very popular in catalyst research [2-4]. Derivatives of $6^{\prime}$-Amino-cinchonine 1 and $6^{\prime}$-Amino-cinchonidine 
2, as well as the compounds themselves, could have an interesting place in the addressed fields of medicine and catalysis. With our experience [5-15] in the field of this substance class, we began a project to investigate the feasible chemistry more deeply and, thus, generate potentially interesting compounds. Since our group has experience in the general area of cycloadditions [16] and, in particular, the Husigen [3 + 2] cycloaddition [17], we decided to use this technique for the establishment of a triazole unit. The synthesis plan was to convert the amines into the corresponding azides and then couple them to terminal alkynes in a Husigen [3 + 2] cycloaddition. This well-established chemistry, also known as click chemistry, offers a rich pool of diverse reaction possibilities [18-23] and thus opens up access to this interesting substitution pattern.

\section{Results}

\subsection{Chemistry}

In addition to the aforementioned $6^{\prime}$-Amino-cinchonine $\mathbf{1}$ and $6^{\prime}$-Amino-cinchonidine 2, these also used the vinyl side chain manipulated derivatives (Figure 2). Moreover, the chemistry was carried out on the hydrogenated compounds 3 and 4 as well as on the dehydrogenated derivatives $\mathbf{5}$ and $\mathbf{6}$. In the case of the latter compounds, the question becomes whether inter- or intramolecular [3+2] cycloadditions can be observed.<smiles>C=CC1CN2CCC1C2[C@H](O)c1ccnc2ccc(N)cc12</smiles>

6'-Amino-cinchonine 1<smiles>C=CC1CN2CCC1C2[C@H](O)c1ccnc2ccc(N)cc12</smiles>

6'-Amino-cinchonidine 2<smiles>CCC1CN2CCC1C2[C@H](O)c1ccnc2ccc(N)cc12</smiles>

6'-Amino-10,11-dihydrocinchonine 3<smiles>CCC1CN2CCC1C[C@H]2[C@H](O)c1ccnc2ccc(N)cc12</smiles>

6'-Amino-10,11-dihydrocinchonidine 4<smiles>C#CC1CN2CCC1CN2[C@H](O)c1ccnc2ccc(N)cc12</smiles>

6'-Amino-10,11-didehydrocinchonine 5<smiles>C#CC1CN2CCC1C[C@@H]2[C@H](O)c1ccnc2ccc(N)cc12</smiles>

6'-Amino-10,11-didehydrocinchondiine 6

Figure 2. Starting materials: $6^{\prime}$-Amino-cinchonine 1 and $6^{\prime}$-Amino-cinchonidine 2 , plus their hydrogenated derivatives ( 3 and 4 ) and their dehydrogenated derivatives ( 5 and 6 ).

\subsubsection{Introduction of the Azido Functionality}

Azide functionality is introduced by a diazotization protocol [24]. Scheme 1 details the conversion of amine $\mathbf{1}$ to the azido derivative $\mathbf{8}$. In the first step, the amino group is converted into the diazo group under acidic conditions and the intermediate diazonium species $\mathbf{7}$ is formed. Without intermediate work-up, the diazo group is substituted by addition of sodium azide to form the $6^{\prime}$-azido-cinchonine 8 in good yield (89\%) and purity. The protocol could also be successfully applied to amines $\mathbf{2}$ to $\mathbf{6}$ with similarly good yields (Table 1). Under the conditions chosen, alkynes 5 and $\mathbf{6}$ also gave no indication of any recognizable spontaneous triazole ring closing. The yields were within the range of the other conversions. 


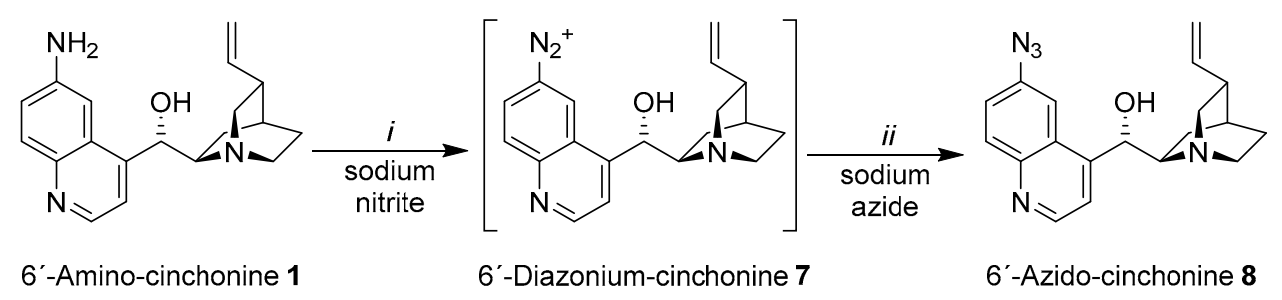

i: $\mathrm{NaNO}_{2}, \mathrm{HCl}(15 \%),-5^{\circ} \mathrm{C}, 1 \mathrm{~h}$; ii: $\mathrm{NaN}_{3}$, water, $0^{\circ} \mathrm{C}, 2 \mathrm{~h}$; yield over both steps: $89 \%$.

Scheme 1. Synthesis of 6'-Azido-cinchonine 8, starting from $6^{\prime}$-Amino-cinchonine 1 via the diazonium species 7.

Table 1. Yields of the transformation of the amins 2 to 6 to the corresponding azides 9 to 13 .

\begin{tabular}{|c|c|c|}
\hline $\begin{array}{l}\text { Amino Compound } \\
\text { (Starting Material) }\end{array}$ & $\begin{array}{l}\text { Azido Compound } \\
\text { (Product) }\end{array}$ & Yield \\
\hline 6'-Amino-cinchonidine 2 & 6'-Azido-cinchonidine 9 & $96 \%$ \\
\hline Dihydro-6'-Amino-cinchonine 3 & Dihydro-6'-Azido-cinchonine 10 & $78 \%$ \\
\hline Dihydro-6'-Amino-cinchonidine 4 & Dihydro-6'-Azido-cinchonidine $\mathbf{1 1}$ & $79 \%$ \\
\hline Didehydro-6 $6^{\prime}$-Amino-cinchonine 5 & Didehydro-6'-Azido-cinchonine 12 & $97 \%$ \\
\hline Didehydro- $6^{\prime}$-Amino-cinchonidine 6 & Didehydro- 6 -Azido-cinchonidine $\mathbf{1 3}$ & $94 \%$ \\
\hline
\end{tabular}

To establish triazole functionality, azides $\mathbf{8}$ to $\mathbf{1 1}$ were reacted with trimethylsilylacetylene according to the protocol of Zhao et al. [18]. The synthesis protocol originally provided a method in which the cleavage of the silyl group is carried out in situ. In order to see the orientation of the Husigen [3 + 2] cycloaddition, the reaction was carried out in two steps. The silylated species was isolated and characterized. As shown in Scheme 2, only the triazole 14 silylated in position 4 is formed from the azide 8 in a yield of $90 \%$. This was clearly detected by NMR experiments. In a final step, compound $\mathbf{1 4}$ was converted to the unsubstituted triazole 15 via E1cb mechanism. Here the yield was $81 \%$.

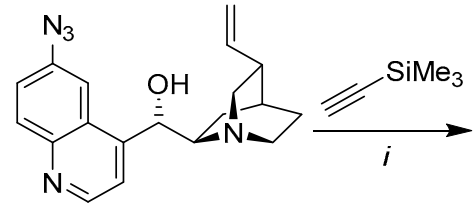

6'-Azido-cinchonine 8

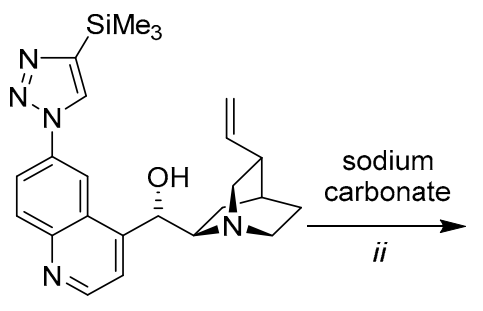

$6^{\prime}$-(4-Silyl-triazolo)cinchonine 14<smiles>C=CC1CC2CCN1C2[C@H](O)c1ccnc2ccc(-n3cc(C)nn3)cc12</smiles>

6'-Triazolo-cinchonine 15

i: $\mathrm{Cu}_{2} \mathrm{SO}_{4}$, sodium ascorbate, $\mathrm{MeOH}$, water, $20^{\circ} \mathrm{C}, 24 \mathrm{~h}, 90 \%$; ii: $\mathrm{K}_{2} \mathrm{CO}_{3}, t-\mathrm{BuOH}$, water, $20^{\circ} \mathrm{C}, 48 \mathrm{~h}, 81 \%$.

Scheme 2. Synthesis of $6^{\prime}$-Triazlo-cinchonine 15, starting from $6^{\prime}$-Azido-cinchonine 8 . The intermediate was the silylated compound $\mathbf{1 4}$, isolated and characterized.

\subsubsection{Assembling of the Triazole Unit}

Azides 9, 10 and $\mathbf{1 1}$ were converted into the corresponding silylated triazoles 16, 18 and 20 and monosubstituted triazoles 17, 19 and 21 using the same protocol. The yields ranged from good to excellent ( 89 to $94 \%$ for the cyclo-addition and $81-87 \%$ for the desilylation).

\subsubsection{Assembling of the Triazole Unit Containing a Further Quinuclidine Rest}

After it was shown that the triazole unit can be, in principle, introduced in good yields, more complex molecules were chosen as alkyne sources in a second phase. The alkynes didehydroquincoridine 22 and didehydrocincorine 23 (Figure 3), also compounds derived from the Cinchona alkaloids, were chosen. 


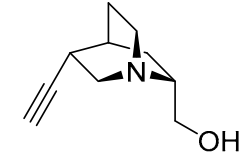

Didehydroquincoridine 22

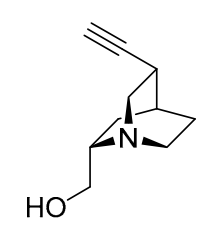

Didehydroquincorine $\mathbf{2 3}$

Figure 3. Didehydroquincoridine 22 and didehydrocincorine 23, Cinchona alkaloid based compounds.

Another protocol by Singh et al. was used [19]. The two alkynes $\mathbf{2 2}$ and $\mathbf{2 3}$ were reacted with the four azides 8-11. Scheme 3 illustrates the two reactions of didehydroquincoridine $\mathbf{2 2}$ with the hydrated azides $\mathbf{1 0}$ and $\mathbf{1 1}$.

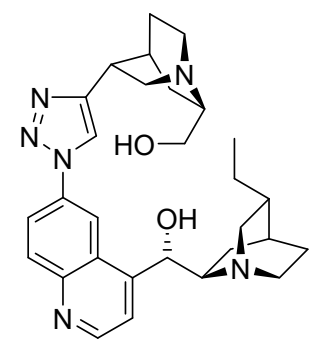

Triazole 28

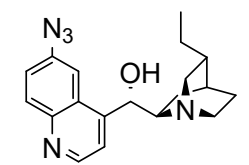

Dihydro-6'-Azidocinchonine 10

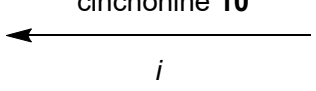

Didehydroquincoridine 22

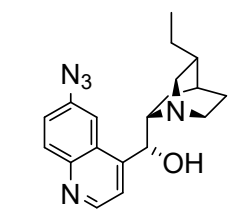

Dihydro-6'-Azidocinchonidine 11 i 22

i: $\mathrm{Cu}_{2} \mathrm{SO}_{4}$, sodium ascorbate, $t-\mathrm{BuOH}$, water, $20^{\circ} \mathrm{C}, 24 \mathrm{~h}$, yields: $65 \%$ for 28 and $72 \%$ for 30 .

Scheme 3. Synthesis of $(S)-(6-(4-((1 S, 3 R, 4 S, 6 R)-6-(h y d r o x y m e t h y l) q u i n u c l i d i n-3-y l)-1 H-1,2,3-t r i a z o l-1-y l) q u i n o l i n-4-$ $\mathrm{yl})((1 S, 2 R, 4 S, 5 R)-5$-ethylquinuclidin-2-yl)methanol (28) and one of its diastereomers 30, starting from azides $\mathbf{1 0}$ and $\mathbf{1 1}$ using didehydroquincoridine 22.

The synthesis of compounds 24 to 31 was again successful in moderate to good yields (62-82\%). Two trends can be observed. The yields in the cinchonidine series (azides 9 and 11, 72-82\%) were better than in the cinchonine series (azides 8 and 10, 62-78\%). Although the difference is not strikingly large, it cannot be ignored. Furthermore, in three pairs, the yields of the hydrogenated compounds were higher than the yields of the corresponding vinyl species.

\subsection{X-ray}

We investigated three of the synthesized compounds by crystal structure analysis: azides 10 and 11 (Figure 4) and the compound 28 (Figure 5). The compounds 10 and 11 crystalized in their anti-open conformer. Crystallographic data collection parameter of 10, $\mathbf{1 1}$ and $\mathbf{2 8}$ can be found in the Supplementary Matericals.

\subsection{IR Spectroscopy, Melting Point and NMR Experiments}

The substances were investigated by infrared spectroscopy. Azides $\mathbf{8}$ to $\mathbf{1 3}$ showed a characteristic band at a wavenumber of $v=2104$ to $2108 \mathrm{~cm}^{-1}$. The silylated triazoles 14, 16, 18 and 20 had a prominent band at a wavenumber of $v=837$ to $839 \mathrm{~cm}^{-1}$. When the melting point was determined, all synthesized compounds decomposed and gas evolved. In the ${ }^{1} \mathrm{H}$ - and ${ }^{13} \mathrm{C}$-NMR experiments, the signals were in the range expected for Cinchona alkaloids. Hydroxy protons were not detected at all or only as very broad signals. 


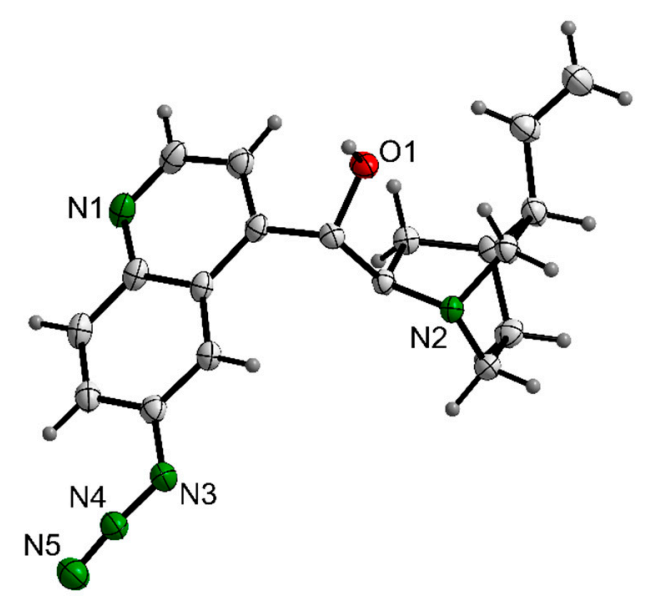

Dihydro-6'-Azido-cinchonine $\mathbf{1 0}$

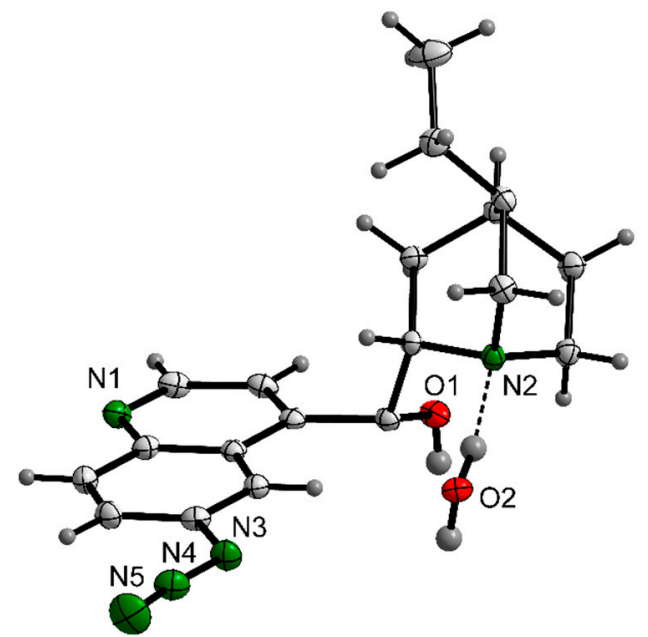

Dihydro-6'-Azido-cinchonidine 11

Figure 4. The X-ray analysis for azide $\mathbf{1 0}$ and $\mathbf{1 1 .}$

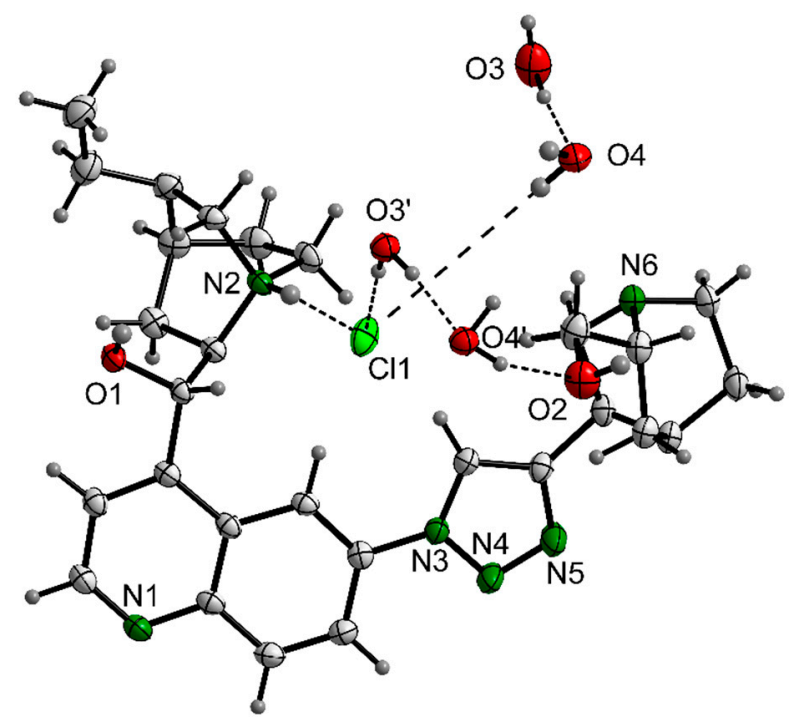

Triazole 28

Figure 5. The X-ray analysis for compound 28.

\subsection{Further Experiments}

We gave special attention to azide-alkyne $\mathbf{1 2}$ in order to perform an intramolecular Husigen [3 + 2] cycloaddition. Simple observations on the model showed that this compound can be built up without significant ring stresses. The only condition is that azide 12 turns into a certain possible conformation, however, this compact molecule eluded our grasp. Protocols $[25,26]$ at elevated temperature led to the formation of amine 5. In dilute solution [21,27], we observed a non-specific decomposition over time. Using the protocols described in this study, there was mass spectroscopic evidence that oligomeric units were formed.

\section{Discussion}

The aim of this work was to investigate the synthetic potential of 6 -Amino-cinchonine 1, 6'-Amino-cinchonidine 2 and their derivatives and whether they could be successfully converted into the azides 8-13. Using the well-established Husigen [3 + 2] cycloaddition method, the construction of the triazoles 14, 16, 18, 20, 24-31 was successfully performed. 
Only one addition product was observed in all cases. According to current knowledge, $[3+2]$ cycloaddition reaction can proceed via single step-mechanism or stepwise mechanism with a zwitterionic/biradical intermediate. The stepwise mechanism was recently documented for a reaction involving different types of three-atom components, including azides [28]. Since the observed products are stereochemically less hindered, it is likely a main reason for the observed stereochemistry. For considered reactions, the mechanism cannot be proposed a priori since no mechanistic studies were made. The formal additionproducts of ethyne to the azide functionality (Triazoles 15, 17, 19, 21) can be generated by desilylation of the compounds $\mathbf{1 4}, \mathbf{1 6}, \mathbf{1 8}, \mathbf{2 0}$. Overall, all reactions proceeded with good purity and good to very good yields. The aim of future work will be to investigate the potential of these compounds in, for example, catalysis or medicinal chemistry. In terms of structural chemistry, the intramolecular cyclisation of azide alkynes 5 and $\mathbf{6}$ is a challenge for every synthetic chemist.

\section{Materials and Methods}

\subsection{Chemistry}

\subsubsection{General Information}

All reagents were purchased from commercial sources (Sigma-Aldrich, TCI, Acros or Buchler) and used without further purification. The solvents used were of analytical grade. The $1 \mathrm{H}$ and 13C NMR spectra were recorded at room temperature on a Bruker Avance 300, Bruker Avance 400 or Bruker Avance 600, operating at $300 \mathrm{MHz}, 400 \mathrm{MHz}$ or $600 \mathrm{MHz}$ for $1 \mathrm{H}$ and $75 \mathrm{MHz}, 100 \mathrm{MHz}$ or $150 \mathrm{MHz}$ for $13 \mathrm{C}$. Chemical shifts ( $\delta$ ) were reported as relative to the tetramethylsilane peak set at $0.00 \mathrm{ppm}$. In the case of multiplets, the signals were reported as intervals. The signals were abbreviated as s, singlet; $d$, doublet; $t$, triplet; q, quadruplet; and m, multiplet. Mass spectra were recorded on a Finnigan MAT 8400-MSS and Finnigan MAT 4515. High resolution mass spectra were recorded on a Finnigan MAT $95 \mathrm{XP}$. The reactions were monitored by TLC and performed on silica gel plates $40 \times 80 \mathrm{~mm}$ Polygram Sil G/UV254 (Macherey-Nagel). Visualization on TLC was achieved by UV light. Column chromatography was performed with Merck silica gel 60 (70-200 mesh).

The numbering of the Cinchona core followed the rules of Paul Rabe, with the side chain numbered based on the IUPAC rules.

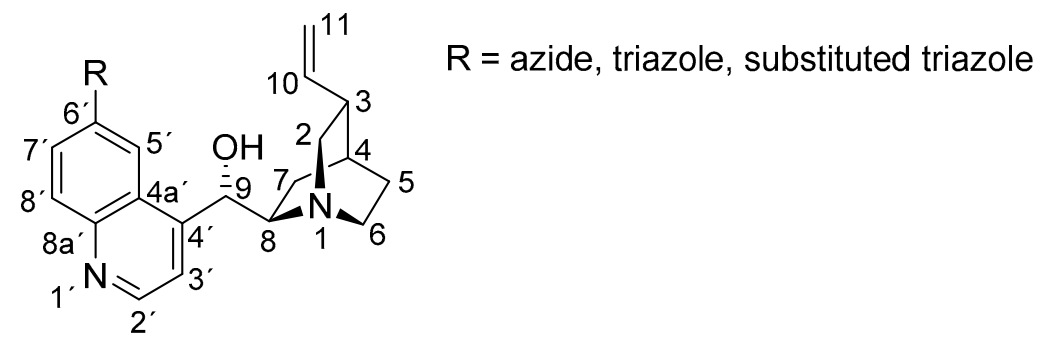

\subsubsection{Synthesis of the Azido-Derivatives 8-13}

General procedure: a dropwise a solution of $\mathrm{NaNO}_{2}(1.2 \mathrm{eq})$ in $\mathrm{H}_{2} \mathrm{O}(0.33 \mathrm{~g} / \mathrm{mL})$ was added to a mixture of Cinchona amine $(1.0 \mathrm{eq})$ and $15 \% \mathrm{HCl}(0.1 \mathrm{~g}$ amine $/ \mathrm{mL})$ cooled at $-5{ }^{\circ} \mathrm{C}$. After the addition was complete, the reaction mixture was stirred at this temperature for 60 min. A solution of $\mathrm{NaN}_{3}(1.7 \mathrm{eq})$ in $\mathrm{H}_{2} \mathrm{O}(0.2 \mathrm{~g} / \mathrm{mL})$ was added dropwise to the reaction mixture at $0{ }^{\circ} \mathrm{C}$. After the addition was finished, the reaction mixture was maintained at $0{ }^{\circ} \mathrm{C}$ for $2 \mathrm{~h}$ and then stirred at room temperature for $12 \mathrm{~h}$. The product was precipitated by addition of $\mathrm{NaOH}(20 \%)$ until $\mathrm{pH} 10$. Upon filtration, the solid was washed with distilled water and dried. Crystallization from DCM presented the Cinchona azides as yellow solids in good yields (70-90\%)

(S)-(6-Azidoquinolin-4-yl)((1S,2R,4S,5R)-5-vinylquinuclidin-2-yl)methanol (8): the title compound was synthesized from $1(25.0 \mathrm{~g}, 81 \mathrm{mmol})$ to afford $8(25.8 \mathrm{~g}$, yield: $95 \%)$ as a yellow-orange solid. ${ }^{1} \mathrm{H}-\mathrm{NMR}(\mathrm{MeOH}-\mathrm{d} 4,400 \mathrm{MHz}) \delta 8.79\left(\mathrm{~d}, J=4.6 \mathrm{~Hz}, \mathrm{H}-2^{\prime}\right), 8.08(\mathrm{~d}$, 
$\left.J=9.05 \mathrm{~Hz}, \mathrm{H}-8^{\prime}\right), 7.82\left(\mathrm{~d}, J=2.4 \mathrm{~Hz}, \mathrm{H}-5^{\prime}\right), 7.74\left(\mathrm{~d}, J=4.6 \mathrm{~Hz}, \mathrm{H}-3^{\prime}\right), 7.52(\mathrm{dd}, J=9.05 \mathrm{~Hz}$, $\left.J=2.45 \mathrm{~Hz}, \mathrm{H}-7^{\prime}\right), 6.17(\mathrm{ddd}, J=17.4 \mathrm{~Hz}, J=10.3 \mathrm{~Hz}, J=7.7 \mathrm{~Hz}, \mathrm{H}-10), 5.66-5.52$ (m, H-9), 5.17-5.08 (m, H-11, H-11), 3.50-3.43 (m, H-2), 3.35-3.32 (m, H-8), 2.96-2.74 (m, H-2, H-6, H-6), 2.37-2.29 (m, H-3), 2.23-2.16 (m, H-7), 1.93-1.68 (m, H-4), 1.66-1.51 (m, H-5, H-5), and 1.31-1.18 (m, H-7); ${ }^{13} \mathrm{C}-\mathrm{NMR}(\mathrm{MeOH}-d 4,100 \mathrm{MHz}) \delta 151.27\left(\mathrm{C}_{\mathrm{q}}, \mathrm{C}-4^{\prime}\right), 150.32\left(\mathrm{CH}, \mathrm{C}-2^{\prime}\right)$, $146.64\left(\mathrm{C}_{\mathrm{q}}, \mathrm{C}-8 \mathrm{a}^{\prime}\right) ; 141.73(\mathrm{CH}, \mathrm{C}-10) ; 140.31\left(\mathrm{C}_{\mathrm{q}}, \mathrm{C}-6^{\prime}\right), 132.14\left(\mathrm{CH} ; \mathrm{C}-8^{\prime}\right), 128.03\left(\mathrm{C}_{\mathrm{q}}, \mathrm{C}-4 \mathrm{a}^{\prime}\right)$, $123.87\left(\mathrm{CH}, \mathrm{C}-7^{\prime}\right), 120.87\left(\mathrm{CH} ; \mathrm{C}-3^{\prime}\right), 115.15\left(\mathrm{CH}_{2}, \mathrm{C}-11\right), 112.90\left(\mathrm{CH}, \mathrm{C}-5^{\prime}\right), 72.49(\mathrm{CH} ; \mathrm{C}-9)$, $61.27(\mathrm{CH}, \mathrm{C}-8), 50.82\left(\mathrm{CH}_{2}, \mathrm{C}-6\right), 50.19\left(\mathrm{CH}_{2}, \mathrm{C}-2\right), 41.29(\mathrm{CH}, \mathrm{C}-3), 29.65(\mathrm{CH}, \mathrm{C}-4), 27.20$ $\left(\mathrm{CH}_{2}, \mathrm{C}-5\right)$, and $22.25\left(\mathrm{CH}_{2}, \mathrm{C}-7\right) ; \mathrm{H}, \mathrm{H}-\mathrm{Cosy}, \mathrm{HSQC}$ and $\mathrm{HMBC}$ were made. Melting point: the substance decomposed at $\mathrm{T}=173{ }^{\circ} \mathrm{C}$; TLC (TBME: $\mathrm{MeOH}$ : ammonia 25\%/100:10:1): rf-value $=0.60$; IR: see spectrum in the supporting information; HR-MS (ESI, MeOH) was calculated for $\mathrm{C}_{19} \mathrm{H}_{21} \mathrm{~N}_{5} \mathrm{O}_{1}+\mathrm{H}^{+}$: $336.18189 \mathrm{Da}$, found 336.18185 Da.

(R)-(6-Azidoquinolin-4-yl)((1S,2S,4S,5R)-5-vinylquinuclidin-2-yl)methanol (9): the title compound was synthesized from $2(25.0 \mathrm{~g}, 81 \mathrm{mmol})$ to afford $9(24.1 \mathrm{~g}$, yield: $89 \%)$ as a yellow-orange solid. ${ }^{1} \mathrm{H}-\mathrm{NMR}(\mathrm{MeOH}-d 4,400 \mathrm{MHz}) \delta 8.76\left(\mathrm{~d}, J=4.57 \mathrm{~Hz}, \mathrm{H}-2^{\prime}\right), 8.05(\mathrm{~d}$, $\left.J=9.05 \mathrm{~Hz}, \mathrm{H}-8^{\prime}\right), 7.82\left(\mathrm{~d}, J=2.4 \mathrm{~Hz}, \mathrm{H}-5^{\prime}\right), 7.71\left(\mathrm{~d}, J=4.57 \mathrm{~Hz}, \mathrm{H}-3^{\prime}\right), 7.52(\mathrm{dd}, J=9.05 \mathrm{~Hz}$, $\left.J=2.4 \mathrm{~Hz}, \mathrm{H}-7^{\prime}\right), 5.78(\mathrm{ddd}, J=17.7 \mathrm{~Hz}, J=10.7 \mathrm{~Hz}, J=7.7 \mathrm{~Hz}, \mathrm{H}-10), 5.53-5.49$ (m, H-9), 5.02-4.94 (m, H-11, H-11), 3.61-3.51 (m, H-6), 3.12-2.94 (m, H-2, H-8), 2.72-2.49 (m, H-2, $\mathrm{H}-6), 2.48-2.18$ (m; H-3), 1.93-1.81 (m,H-5, H-7), 1.80-1.78 (m, H-4), and 1.72-1.50 (m, H-5, H-7); ${ }^{13} \mathrm{C}-\mathrm{NMR}(\mathrm{MeOH}-d 4,100 \mathrm{MHz}) \delta 151.22\left(\mathrm{C}_{\mathrm{q}}, \mathrm{C}-4^{\prime}\right), 150.27\left(\mathrm{CH}, \mathrm{C}-2^{\prime}\right), 146.62\left(\mathrm{C}_{\mathrm{q}}\right.$, C-8a'); $142.78(\mathrm{CH}, \mathrm{C}-10) ; 140.24\left(\mathrm{C}_{\mathrm{q}}, \mathrm{C}-6^{\prime}\right), 132.15\left(\mathrm{CH}, \mathrm{C}-8^{\prime}\right), 127.95\left(\mathrm{C}_{\mathrm{q}}, \mathrm{C}-4 \mathrm{a}^{\prime}\right), 123.25$ $\left(\mathrm{CH}, \mathrm{C}-7^{\prime}\right), 120.93\left(\mathrm{CH}, \mathrm{C}-3^{\prime}\right), 114.90\left(\mathrm{CH}_{2}, \mathrm{C}-11\right), 112.99\left(\mathrm{CH}, \mathrm{C}-5^{\prime}\right), 72.40(\mathrm{CH}, \mathrm{C}-9), 61.53$ $(\mathrm{CH}, \mathrm{C}-8), 57.59\left(\mathrm{CH}_{2}, \mathrm{C}-2\right), 43.82\left(\mathrm{CH}_{2}, \mathrm{C}-6\right), 40.95(\mathrm{CH}, \mathrm{C}-3), 29.18(\mathrm{CH}, \mathrm{C}-4), 28.25\left(\mathrm{CH}_{2}\right.$, $\mathrm{C}-5)$, and $22.61\left(\mathrm{CH}_{2}, \mathrm{C}-7\right) ; \mathrm{H}, \mathrm{H}-\mathrm{Cosy}, \mathrm{HSQC}$ and HMBC were made. Melting point: the substance decomposed at $\mathrm{T}=144{ }^{\circ} \mathrm{C}$; TLC (TBME: MeOH: ammonia 25\%/100:10:1): rfvalue $=0.62$; IR: see spectrum in the supporting information; HR-MS (ESI, MeOH) was calculated for $\mathrm{C}_{19} \mathrm{H}_{21} \mathrm{~N}_{5} \mathrm{O}_{1}+\mathrm{H}^{+}$: $336.18189 \mathrm{Da}$, found 336.18183 Da.

(S)-(6-Azidoquinolin-4-yl)((1S,2R,4S,5R)-5-ethylquinuclidin-2-yl)methanol (10): the title compound was synthesized from $3(5.0 \mathrm{~g}, 16.0 \mathrm{mmol})$ to afford $10(4.3 \mathrm{~g}$, yield: $79 \%)$ as a yellowish solid. ${ }^{1} \mathrm{H}-\mathrm{NMR}$ (DMSO- $\left.d 6,400 \mathrm{MHz}\right) \delta 8.78\left(\mathrm{~d}, J=4.5 \mathrm{~Hz}, \mathrm{H}-2^{\prime}\right), 8.04(\mathrm{~d}$, $\left.J=9.0 \mathrm{~Hz}, \mathrm{H}-8^{\prime}\right), 7.86\left(\mathrm{~d}, J=2.5 \mathrm{~Hz}, \mathrm{H}-5^{\prime}\right), 7.54\left(\mathrm{~d}, J=4.5 \mathrm{~Hz}, \mathrm{H}-3^{\prime}\right), 7.48(\mathrm{dd}, J=9.0 \mathrm{~Hz}$, $\left.J=2.5 \mathrm{~Hz}, \mathrm{H}-7^{\prime}\right), 5.20-5.16$ (m, H-9), 2.96-2.88 (m, H-8), 2.68-2.62 (m, H-6, H-6), 2.52-2.44 (m, H-2, H-2, H-3), 2.00-2.76 (m, H-7), 1.65-1.62 (m, H-4); 1.53-1.30 (m, H-5, H-5, H-7, $\mathrm{H}-10, \mathrm{H}-10)$, and 0.86 (t, $J=7.2 \mathrm{~Hz}, \mathrm{H}-11, \mathrm{H}-11, \mathrm{H}-11) ;{ }^{13} \mathrm{C}-\mathrm{NMR}$ (DMSO-d6, $100 \mathrm{MHz}$ ) $\delta$ $150.30\left(\mathrm{C}_{\mathrm{q}}, \mathrm{C}-4^{\prime}\right), 149.50\left(\mathrm{CH}, \mathrm{C}-2^{\prime}\right), 145.62\left(\mathrm{C}_{\mathrm{q}}, \mathrm{C}-8 \mathrm{a}^{\prime}\right), 136.85\left(\mathrm{C}_{\mathrm{q}}, \mathrm{C}-6^{\prime}\right), 131.70\left(\mathrm{CH}, \mathrm{C}-8^{\prime}\right)$, $126.94\left(\mathrm{C}_{\mathrm{q}}, \mathrm{C}-4 \mathrm{a}^{\prime}\right), 121.56\left(\mathrm{CH}, \mathrm{C}-7^{\prime}\right), 119.76\left(\mathrm{CH}, \mathrm{C}-3^{\prime}\right), 112.15\left(\mathrm{CH}, \mathrm{C}-5^{\prime}\right), 70.73(\mathrm{CH}, \mathrm{C}-9)$, $61.14(\mathrm{CH}, \mathrm{C}-8), 49.89\left(\mathrm{CH}_{2}, \mathrm{C}-6\right), 49.11\left(\mathrm{CH}_{2}, \mathrm{C}-2\right), 37.08(\mathrm{CH}, \mathrm{C}-3), 27.11\left(\mathrm{CH}_{2}, \mathrm{C}-5\right), 26.01$ $(\mathrm{CH}, \mathrm{C}-4), 25.03\left(\mathrm{CH}_{2}, \mathrm{C}-10\right), 24.01\left(\mathrm{CH}_{2}, \mathrm{C}-7\right)$, and $11.90\left(\mathrm{CH}_{3}, \mathrm{C}-11\right) ; \mathrm{H}, \mathrm{H}-\mathrm{Cosy}, \mathrm{HSQC}$ and HMBC were made. Melting point: the substance decomposed at $\mathrm{T}=191{ }^{\circ} \mathrm{C}$; TLC (TBME: $\mathrm{MeOH}$ : ammonia 25\%/100:10:1): rf-value $=0.50$; IR: see spectrum in the supporting information; HR-MS (ESI, MeOH) calculated for $\mathrm{C}_{19} \mathrm{H}_{23} \mathrm{~N}_{5} \mathrm{O}_{1}+\mathrm{H}^{+}$: $338.19754 \mathrm{Da}$, found 338.19756 Da.

(R)-(6-Azidoquinolin-4-yl)((1S,2S,4S,5R)-5-ethylquinuclidin-2-yl)methanol (11): the title compound was synthesized from $4(5.0 \mathrm{~g}, 16 \mathrm{mmol})$ to afford $8(4.2 \mathrm{~g}$, yield: $78 \%)$ as a yellow solid. ${ }^{1} \mathrm{H}-\mathrm{NMR}$ (DMSO- $\left.d 6,400 \mathrm{MHz}\right) \delta 8.78\left(\mathrm{~d}, J=4.45 \mathrm{~Hz}, \mathrm{H}-2^{\prime}\right), 8.05\left(\mathrm{~d}, J=9.0 \mathrm{~Hz}, \mathrm{H}-8^{\prime}\right)$, $7.91\left(\mathrm{~d}, J=2.4 \mathrm{~Hz}, \mathrm{H}-5^{\prime}\right), 7.55\left(\mathrm{~d}, J=4.45 \mathrm{~Hz}, \mathrm{H}-3^{\prime}\right), 7.51\left(\mathrm{dd}, J=9.0 \mathrm{~Hz}, J=2.4 \mathrm{~Hz}, \mathrm{H}-7^{\prime}\right)$, 5.18-5.13 (m, H-9), 3.21-2.98 (m, H-6, H-8), 2.87-2.77 (m, H-2), 2.45-2.31 (m, H-6), 2.18-2.06 (m, H-2), 1.71-1.50 (m; H-4, H-5, H-7, H-7), 1.40-1.15 (m, H-3, H-5, H-10, H-10), and 0.86 (t, $J=7.1 \mathrm{~Hz}, \mathrm{H}-11, \mathrm{H}-11, \mathrm{H}-11) ;{ }^{13} \mathrm{C}-\mathrm{NMR}$ (DMSO-d6, $\left.100 \mathrm{MHz}\right) \delta 149.87$ (CH, C-2'), 149.50 $\left(\mathrm{C}_{\mathrm{q}}, \mathrm{C}-4^{\prime}\right), 145.75\left(\mathrm{C}_{\mathrm{q}}, \mathrm{C}-8 \mathrm{a}^{\prime}\right) ; 136.92\left(\mathrm{C}_{\mathrm{q}}, \mathrm{C}-6^{\prime}\right), 131.79\left(\mathrm{CH}, \mathrm{C}-8^{\prime}\right), 126.87\left(\mathrm{C}_{\mathrm{q}}, \mathrm{C}-4 \mathrm{a}^{\prime}\right), 121.45$ $\left(\mathrm{CH}, \mathrm{C}-7^{\prime}\right), 119.97\left(\mathrm{CH}, \mathrm{C}-3^{\prime}\right), 112.73\left(\mathrm{CH}, \mathrm{C}-5^{\prime}\right), 71.44(\mathrm{CH}, \mathrm{C}-9), 60.59(\mathrm{CH}, \mathrm{C}-8), 57.53\left(\mathrm{CH}_{2}\right.$, $\mathrm{C}-2), 41.64\left(\mathrm{CH}_{2}, \mathrm{C}-6\right), 37.14(\mathrm{CH}, \mathrm{C}-3), 28.18\left(\mathrm{CH}_{2}, \mathrm{C}-5\right), 27.20\left(\mathrm{CH}_{2}, \mathrm{C}-10\right), 25.14(\mathrm{CH}$, $\mathrm{C}-4), 24.35\left(\mathrm{CH}_{2}, \mathrm{C}-7\right)$, and $12.04\left(\mathrm{CH}_{3}, \mathrm{C}-11\right) ; \mathrm{H}, \mathrm{H}-\mathrm{Cosy}, \mathrm{HSQC}$ and $\mathrm{HMBC}$ were made. 
Melting point: the substance decomposed at $\mathrm{T}=163^{\circ} \mathrm{C}$; $\mathrm{TLC}$ (TBME: MeOH: ammonia 25\%/100:10:1): rf-value $=0.54$; IR: see spectrum in the supporting information; HR-MS (ESI, MeOH) calculated for $\mathrm{C}_{19} \mathrm{H}_{23} \mathrm{~N}_{5} \mathrm{O}_{1}+\mathrm{H}^{+}$: 338.19754 Da, found 338.19757 Da.

(S)-(6-Azidoquinolin-4-yl)((1S,2R,4S,5S)-5-ethynylquinuclidin-2-yl)methanol (12): the title compound was synthesized from $5(1.7 \mathrm{~g}, 5.5 \mathrm{mmol})$ to afford $12(1.8 \mathrm{~g}$, yield: $97 \%)$ as a yellow solid. ${ }^{1} \mathrm{H}-\mathrm{NMR}\left(\mathrm{CDCl}_{3}, 300 \mathrm{MHz}\right) \delta 8.73\left(\mathrm{~d}, J=4.5 \mathrm{~Hz}, \mathrm{H}-2^{\prime}\right), 8.07\left(\mathrm{~d}, J=9.0 \mathrm{~Hz}, \mathrm{H}-8^{\prime}\right)$, $7.68\left(\mathrm{~d}, J=2.3 \mathrm{~Hz}, \mathrm{H}-5^{\prime}\right), 7.53\left(\mathrm{~d}, J=4.5 \mathrm{~Hz}, \mathrm{H}-3^{\prime}\right), 7.36\left(\mathrm{dd}, J=9.0 \mathrm{~Hz}, J=2.3 \mathrm{~Hz}, \mathrm{H}-7^{\prime}\right)$, 5.58-5.48 (m, H-9), 3.28-3.20 (m, H-2), 3.18-3.09 (m, H-8), 3.03-2.94 (m, H-2), 2.84-2.74 (m, H-6), 2.68-2.55 (m, H-6), 2.51-2.44 (m, H-3), 2.28-2.16 (m, H-7), 2.15 (d, J = 2.45 Hz, H-11), 2.07-1.93 (m, H-4), and 1.60-1.41 (m, H-5, H-5, H-7); ${ }^{13} \mathrm{C}-\mathrm{NMR}\left(\mathrm{CDCl}_{3}, 75 \mathrm{MHz}\right) \delta 149.50$ $\left(\mathrm{CH}, \mathrm{C}-2^{\prime}\right), 147.93\left(\mathrm{C}_{\mathrm{q}}, \mathrm{C}-4^{\prime}\right), 146.11\left(\mathrm{C}_{\mathrm{q}}, \mathrm{C}-8 \mathrm{a}^{\prime}\right), 138.16\left(\mathrm{C}_{\mathrm{q}}, \mathrm{C}-6^{\prime}\right), 132.22\left(\mathrm{CH}, \mathrm{C}-8^{\prime}\right), 126.81$ $\left(\mathrm{C}_{\mathrm{q}}, \mathrm{C}-4 \mathrm{a}^{\prime}\right), 121.60\left(\mathrm{CH}, \mathrm{C}-7^{\prime}\right), 119.47\left(\mathrm{CH}, \mathrm{C}-3^{\prime}\right), 111.73\left(\mathrm{CH}, \mathrm{C}-5^{\prime}\right), 87.31(\mathrm{CH}, \mathrm{C}-11), 72.01$ $(\mathrm{CH}, \mathrm{C}-9), 69.31\left(\mathrm{C}_{\mathrm{q}}, \mathrm{C}-10\right), 60.25(\mathrm{CH}, \mathrm{C}-8), 50.28\left(\mathrm{CH}_{2}, \mathrm{C}-6\right), 49.41\left(\mathrm{CH}_{2}, \mathrm{C}-2\right), 28.03(\mathrm{CH}$, $\mathrm{C}-3), 27.86(\mathrm{CH}, \mathrm{C}-4), 25.11\left(\mathrm{CH}_{2}, \mathrm{C}-5\right)$, and $23.51\left(\mathrm{CH}_{2}, \mathrm{C}-7\right) ; \mathrm{H}, \mathrm{H}-\mathrm{Cosy}, \mathrm{HSQC}$ and HMBC were made. Melting point: the substance decomposed at $\mathrm{T}=112{ }^{\circ} \mathrm{C}$; $\mathrm{TLC}$ (TBME: $\mathrm{MeOH}$ : ammonia 25\%/100:10:1): rf-value $=0.55$; IR: see spectrum in the supporting information; HR-MS (ESI, MeOH) calculated for $\mathrm{C}_{19} \mathrm{H}_{19} \mathrm{~N}_{5} \mathrm{O}_{1}+\mathrm{H}^{+}$: $334.16624 \mathrm{Da}$, found 334.16647 Da.

(R)-(6-Azidoquinolin-4-yl)((1S,2S,4S,5S)-5-ethynylquinuclidin-2-yl)methanol (13): the title compound was synthesized from $6(1.7 \mathrm{~g}, 5.5 \mathrm{mmol})$ to afford $13(1.4 \mathrm{~g}$, yield: $76 \%)$ as a yellow solid. ${ }^{1} \mathrm{H}-\mathrm{NMR}\left(\mathrm{CDCl}_{3}, 300 \mathrm{MHz}\right) \delta 8.74\left(\mathrm{~d}, J=4.5 \mathrm{~Hz}, \mathrm{H}-2^{\prime}\right), 8.06\left(\mathrm{~d}, J=9.0 \mathrm{~Hz}, \mathrm{H}-8^{\prime}\right)$, $7.95\left(\mathrm{~d}, J=2.5 \mathrm{~Hz}, \mathrm{H}-5^{\prime}\right), 7.51\left(\mathrm{~d}, J=4.5 \mathrm{~Hz}, \mathrm{H}-3^{\prime}\right), 7.21\left(\mathrm{dd}, J=9.0 \mathrm{~Hz}, J=2.5 \mathrm{~Hz}, \mathrm{H}-7^{\prime}\right)$, 5.61-5.55 (m, H-9), 3.42-3-3.34 (m, H-6), 3.24-3.16 (m, H-8), 2.98-2.88 (m, H-2), 2.75-2.61 (m, H-6), 2.61-2.48 (m, H-2), 2.44-2.37 (m, H-3), 2.16 (d, J = 2.4 Hz, H-11), 2.06-1.94 (m,H-5, $\mathrm{H}-7), 1.90-1.89$ (m, H-4), and 1.81-1.59 (m, H-5, H-7); ${ }^{13} \mathrm{C}-\mathrm{NMR}\left(\mathrm{CDCl}_{3}, 75 \mathrm{MHz}\right) \delta 149.65$ $\left(\mathrm{CH}, \mathrm{C}-2^{\prime}\right), 148.02\left(\mathrm{C}_{\mathrm{q}}, \mathrm{C}-4^{\prime}\right), 146.31\left(\mathrm{C}_{\mathrm{q}}, \mathrm{C}-8 \mathrm{a}^{\prime}\right), 138.27\left(\mathrm{C}_{\mathrm{q}}, \mathrm{C}-6^{\prime}\right), 131.97\left(\mathrm{CH}, \mathrm{C}-8^{\prime}\right), 126.86$ $\left(\mathrm{C}_{\mathrm{q}}, \mathrm{C}-4 \mathrm{a}^{\prime}\right), 121.53\left(\mathrm{CH}, \mathrm{C}-7^{\prime}\right), 119.67\left(\mathrm{CH}, \mathrm{C}-3^{\prime}\right), 111.68\left(\mathrm{CH}, \mathrm{C}-5^{\prime}\right), 87.26(\mathrm{CH}, \mathrm{C}-11), 71.87$ $(\mathrm{CH}, \mathrm{C}-9), 69.21\left(\mathrm{C}_{\mathrm{q}}, \mathrm{C}-10\right), 60.62(\mathrm{CH}, \mathrm{C}-8), 56.31\left(\mathrm{CH}_{2}, \mathrm{C}-2\right), 49.42\left(\mathrm{CH}_{2}, \mathrm{C}-6\right), 28.17(\mathrm{CH}$, C-3), $27.96(\mathrm{CH}, \mathrm{C}-4), 24.94\left(\mathrm{CH}_{2}, \mathrm{C}-5\right)$, and $23.47\left(\mathrm{CH}_{2}, \mathrm{C}-7\right) ; \mathrm{H}, \mathrm{H}-\mathrm{Cosy}, \mathrm{HSQC}$ and HMBC were made. Melting point: the substance decomposed at $\mathrm{T}=96{ }^{\circ} \mathrm{C}$; TLC (TBME: $\mathrm{MeOH}$ : ammonia 25\%/100:10:1): rf-value $=0.57$; IR: see spectrum in the supporting information; $H R-M S$ (ESI, MeOH) calculated for $\mathrm{C}_{19} \mathrm{H}_{19} \mathrm{~N}_{5} \mathrm{O}_{1}+\mathrm{H}^{+}: 334.16624 \mathrm{Da}$, found $334.16633 \mathrm{Da}$.

\subsubsection{Synthesis of the Silylated Triazole-Derivatives 14, 16, 18 and 20}

General procedure: in a round-bottom flask the corresponding Cinchona azide (1 eq.) and trimethylsilyl acetylene (1.5 eq.) were suspended in an $\mathrm{H}_{2} \mathrm{O}: \mathrm{MeOH}(1: 1)$ mixture to deliver a substrate concentration of $0.1 \mathrm{M} \mathrm{Cu}_{2} \mathrm{SO}_{4}(0.2 \mathrm{eq}$., $20 \mathrm{~mol} \%)$ and sodium ascorbate ( 0.4 eq., $40 \mathrm{~mol} \%$ ). The reaction mixture was stirred at room temperature until complete conversion of the azide (12-24 h). After that, the reaction was quenched by adding an aqueous solution of ammonium chloride and ammonium hydroxide ( $\mathrm{pH} \mathrm{10/12).} \mathrm{The}$ mixture was extracted with DCM (three times), and the combined organic layers were dried over anhydrous sodium sulphate, filtered and concentrated in vacuo. The residues obtained were purified by flash column chromatography using MTBE: $\mathrm{MeOH}$ as a gradient elution to afford the corresponding TMS-triazole derivatives.

(S)-(6-(4-(Trimethylsilyl)-1H-1,2,3-triazol-1-yl)quinolin-4-yl)((1S,2R,4S,5R)-5-vinylquinuclidin2-yl)methanol (14): the title compound was synthesized from $8(1.0 \mathrm{~g}, 3.0 \mathrm{mmol})$ to afford 14 (1.15 g, yield: $90 \%)$ as a colourless wax. ${ }^{1} \mathrm{H}-\mathrm{NMR}\left(\mathrm{CDCl}_{3}, 300 \mathrm{MHz}\right) \delta 8.77(\mathrm{bs}, \mathrm{H}-$ Triazole-5), 8.49 (d, J = 4.4 Hz, H-2'), 8.10-8.06 (m, H-5' , H-7' $), 7.87$ (d, J = 9.7 Hz, H-8' $), 7.35$ (d, $\left.J=4.4 \mathrm{~Hz}, \mathrm{H}-3^{\prime}\right), 6.32$ (bs, H-9), 6.06 (ddd, $J=17.5 \mathrm{~Hz}, J=10.1 \mathrm{~Hz}, J=7.4 \mathrm{~Hz}, \mathrm{H}-10$ ), 5.24-5.18 (m, H-11, H-11), 4.22-3.96 (m, H-2), 3.34-3.08 (m, H-2, H-8), 3.05-2.88 (m, H-6), 2.58-2.42 (m, H-3), 2.37-2.21 (m, H-6), 1.96-1.87 (m, H-4), 1.85-1.71 (m, H-7), 1.69-1.54 (m, $\mathrm{H}-5), 1.15-1.00(\mathrm{~m}, \mathrm{H}-5)$, and 0.93-0.79 (m, H-7) $0.46\left(\mathrm{~s}, 9 \mathrm{H},-\mathrm{Si}\left(\mathrm{CH}_{3}\right)_{3}\right) ;{ }^{13} \mathrm{C}-\mathrm{NMR}\left(\mathrm{CDCl}_{3}\right.$, $75 \mathrm{MHz}) \delta 150.13\left(\mathrm{CH}, \mathrm{C}-2^{\prime}\right), 147.41\left(\mathrm{C}_{\mathrm{q}}, \mathrm{C}-4^{\prime}\right), 146.93\left(\mathrm{C}_{\mathrm{q}}, \mathrm{C}-\right.$ Triazole-4), $146.62\left(\mathrm{C}_{\mathrm{q}}, \mathrm{C}-8 \mathrm{a}^{\prime}\right)$; $137.55(\mathrm{CH}, \mathrm{C}-10) ; 134.68\left(\mathrm{C}_{\mathrm{q}}, \mathrm{C}^{-6}{ }^{\prime}\right), 131.88\left(\mathrm{CH} ; \mathrm{C}-8^{\prime}\right), 128.35(\mathrm{CH}, \mathrm{C}-$ Triazole-5), 124.52 
$\left(\mathrm{C}_{\mathrm{q}}, \mathrm{C}-4 \mathrm{a}^{\prime}\right), 121.47\left(\mathrm{CH}, \mathrm{C}-7^{\prime}\right), 119.27\left(\mathrm{CH} ; \mathrm{C}-3^{\prime}\right), 116.78\left(\mathrm{CH}_{2}, \mathrm{C}-11\right), 110.91\left(\mathrm{CH}, \mathrm{C}-5^{\prime}\right)$, 67.67 (CH, C-9), $60.37(\mathrm{CH}, \mathrm{C}-8), 49.61\left(\mathrm{CH}_{2}, \mathrm{C}-6\right), 48.71\left(\mathrm{CH}_{2}, \mathrm{C}-2\right), 38.28(\mathrm{CH}, \mathrm{C}-3), 27.67$ $(\mathrm{CH}, \mathrm{C}-4), 24.31\left(\mathrm{CH}_{2}, \mathrm{C}-5\right), 19.00\left(\mathrm{CH}_{2}, \mathrm{C}-7\right)$, and $-1.06\left(\mathrm{CH}_{3},-\mathrm{SiMe} 3\right) ; \mathrm{H}, \mathrm{H}-\mathrm{Cosy}, \mathrm{HSQC}$ and $\mathrm{HMBC}$ were made. Melting point: the substance decomposed at $\mathrm{T}=147^{\circ} \mathrm{C}$; TLC (TBME: $\mathrm{MeOH}$ : ammonia 25\%/100:10:1): rf-value $=0.19$; IR: see spectrum in the supporting information; HR-MS (ESI, MeOH) calculated for $\mathrm{C}_{24} \mathrm{H}_{31} \mathrm{~N}_{5} \mathrm{O}_{1} \mathrm{Si}_{1}+\mathrm{H}^{+}$: $434.23706 \mathrm{Da}$, found 434.23733 Da.

(R)-(6-(4-(Trimethylsilyl)-1H-1,2,3-triazol-1-yl)quinolin-4-yl)((1S,2S,4S,5R)-5-vinylquinuclidin2-yl)methanol (16): the title compound was synthesized from $9(1.0 \mathrm{~g}, 3.0 \mathrm{mmol})$ to afford 16 $(1.2 \mathrm{~g}$, yield: $92 \%)$ as a colourless wax. ${ }^{1} \mathrm{H}-\mathrm{NMR}\left(\mathrm{CDCl}_{3}, 300 \mathrm{MHz}\right) \delta 8.61(\mathrm{~d}, J=4.4 \mathrm{~Hz}$, H-2' $), 8.34$ (bs, H-Triazole-5), $8.28\left(\mathrm{~d}, J=1.6 \mathrm{~Hz}, \mathrm{H}-5^{\prime}\right), 7.97\left(\mathrm{~d}, J=9.1 \mathrm{~Hz}, \mathrm{H}-8^{\prime}\right), 7.90(\mathrm{dd}$, $\left.J=9.1 \mathrm{~Hz}, J=1.6 \mathrm{~Hz}, \mathrm{H}-7^{\prime}\right), 7.52\left(\mathrm{~d}, J=4.4 \mathrm{~Hz}, \mathrm{H}-3^{\prime}\right), 5.87-5.75$ (m, H-9), 5.69 (ddd, $J=17.5$ $\mathrm{Hz}, J=10.3 \mathrm{~Hz}, J=7.4 \mathrm{~Hz}, \mathrm{H}-10), 5.00-4.85$ (m, H-11, H-11), 3.77-3.57 (m, H-6), 3.17-3.03 (m, H-2, H-8), 2.78-2.62 (m, H-2, H-6), 2.41-2.28 (m; H-3), 1.92-1.79 (m, H-4, H-7), 1.62-1.48 (m, H-5, H-5), 0.92-0.81 (m, H-7) $0.41\left(\mathrm{~s}, 9 \mathrm{H},-\mathrm{Si}\left(\mathrm{CH}_{3}\right)_{3}\right) ;{ }^{13} \mathrm{C}-\mathrm{NMR}\left(\mathrm{CDCl}_{3}, 75 \mathrm{MHz}\right) \delta 150.44$ $\left(\mathrm{CH}, \mathrm{C}-2^{\prime}\right), 149.23\left(\mathrm{C}_{\mathrm{q}}, \mathrm{C}-4^{\prime}\right), 147.56\left(\mathrm{C}_{\mathrm{q}}, \mathrm{C}-\right.$ Triazole-4), $146.96\left(\mathrm{C}_{\mathrm{q}}, \mathrm{C}-8 \mathrm{a}^{\prime}\right) ; 140.20(\mathrm{CH}, \mathrm{C}-10)$; $134.34\left(\mathrm{C}_{\mathrm{q}}, \mathrm{C}-6^{\prime}\right), 131.78\left(\mathrm{CH}, \mathrm{C}-8^{\prime}\right), 127.79\left(\mathrm{CH}, \mathrm{C}-\right.$ Triazole-5), $125.50\left(\mathrm{C}_{\mathrm{q}}, \mathrm{C}-4 \mathrm{a}^{\prime}\right), 121.54$ $\left(\mathrm{CH}, \mathrm{C}-7^{\prime}\right), 119.57\left(\mathrm{CH}, \mathrm{C}-3^{\prime}\right), 115.03\left(\mathrm{CH}_{2}, \mathrm{C}-11\right), 112.93\left(\mathrm{CH}, \mathrm{C}-5^{\prime}\right), 69.83(\mathrm{CH}, \mathrm{C}-9), 60.52$ $(\mathrm{CH}, \mathrm{C}-8), 56.12\left(\mathrm{CH}_{2}, \mathrm{C}-2\right), 43.07\left(\mathrm{CH}_{2}, \mathrm{C}-6\right), 39.15(\mathrm{CH}, \mathrm{C}-3), 29.63(\mathrm{CH}, \mathrm{C}-4), 27.52\left(\mathrm{CH}_{2}\right.$, $\mathrm{C}-5), 21.12\left(\mathrm{CH}_{2}, \mathrm{C}-7\right)$, and $-1.14\left(\mathrm{CH}_{3},-\mathrm{SiMe} 3\right) ; \mathrm{H}, \mathrm{H}-\mathrm{Cosy}, \mathrm{HSQC}$ and HMBC were made. Melting point: the substance decomposed at $\mathrm{T}=113^{\circ} \mathrm{C}$; TLC (TBME: $\mathrm{MeOH}$ : ammonia 25\%/100:10:1): rf-value $=0.15$; IR: see spectrum in the supporting information; HR-MS (ESI, MeOH) calculated for $\mathrm{C}_{24} \mathrm{H}_{31} \mathrm{~N}_{5} \mathrm{O}_{1} \mathrm{Si}_{1}+\mathrm{H}^{+}$: $434.23706 \mathrm{Da}$, found $434.23742 \mathrm{Da}$.

(S)-(6-(4-(Trimethylsilyl)-1H-1,2,3-triazol-1-yl)quinolin-4-yl)((1S,2R,4S,5R)-5-ethylquinuclidin-2yl)methanol (18): the title compound was synthesized from $10(0.5 \mathrm{~g}, 1.5 \mathrm{mmol})$ to afford 18 (0.6 g, yield: $94 \%)$ as a colourless honey-type liquid. ${ }^{1} \mathrm{H}-\mathrm{NMR}\left(\mathrm{CDCl}_{3}, 300 \mathrm{MHz}\right) \delta 8.77$ (bs, H-Triazole-5), 8.47 (d, J = 4.4 Hz, H-2'), 8.13-8.09 (m, H-5', H-7'), $7.81(\mathrm{~d}, J=9.7 \mathrm{~Hz}$, H-8 $\left.{ }^{\prime}\right), 7.34\left(\mathrm{~d}, J=4.4 \mathrm{~Hz}, \mathrm{H}-3^{\prime}\right), 6.38$ (bs, H-9), 4.19-3.92 (m, H-2), 3.28-3.04 (m, H-2, H-8), 3.08-2.91 (m, H-6), 2.52-2.37 (m, H-3), 2.31-2.17 (m, H-6), 1.92-1.85 (m, H-4), 1.81-1.70 (m, H-7), 1.61-1.49 (m, H-5), 1.29-1.15 (m, H-5, H-10, H-10), 0.95-0.83 (m, H-7) 0.79 (t, $J=7.2 \mathrm{~Hz}, \mathrm{H}-11, \mathrm{H}-11, \mathrm{H}-11)$, and $0.42\left(\mathrm{~s}, 9 \mathrm{H},-\mathrm{Si}\left(\mathrm{CH}_{3}\right)_{3}\right),{ }^{13} \mathrm{C}-\mathrm{NMR}\left(\mathrm{CDCl}_{3}, 75 \mathrm{MHz}\right) \delta$ $150.20\left(\mathrm{CH}, \mathrm{C}-2^{\prime}\right), 148.27\left(\mathrm{C}_{\mathrm{q}}, \mathrm{C}-4^{\prime}\right), 147.43\left(\mathrm{C}_{\mathrm{q}}, \mathrm{C}-\right.$ Triazole-4), $146.70\left(\mathrm{C}_{\mathrm{q}}, \mathrm{C}-8 \mathrm{a}^{\prime}\right) ; 134.39\left(\mathrm{C}_{\mathrm{q}}\right.$, C-6' $), 131.74\left(\mathrm{CH} ; \mathrm{C}-8^{\prime}\right), 128.03\left(\mathrm{CH}, \mathrm{C}-\right.$ Triazole-5), $124.98\left(\mathrm{C}_{\mathrm{q}}, \mathrm{C}-4 \mathrm{a}^{\prime}\right), 121.33\left(\mathrm{CH}, \mathrm{C}-7^{\prime}\right)$, $119.33\left(\mathrm{CH} ; \mathrm{C}-3^{\prime}\right), 111.88\left(\mathrm{CH}, \mathrm{C}-5^{\prime}\right), 68.77(\mathrm{CH}, \mathrm{C}-9), 60.33(\mathrm{CH}, \mathrm{C}-8), 50.42\left(\mathrm{CH}_{2}, \mathrm{C}-6\right), 49.67$ $\left(\mathrm{CH}_{2}, \mathrm{C}-2\right), 36.38(\mathrm{CH}, \mathrm{C}-3), 26.88\left(\mathrm{CH}_{2}, \mathrm{C}-10\right), 25.58(\mathrm{CH}, \mathrm{C}-4), 24.69\left(\mathrm{CH}_{2}, \mathrm{C}-5\right), 19.48\left(\mathrm{CH}_{2}\right.$, $\mathrm{C}-7), 11.75\left(\mathrm{CH}_{3}, \mathrm{C}-11\right)$, and $-1.15\left(\mathrm{CH}_{3},-\mathrm{SiMe} 3\right) ; \mathrm{H}, \mathrm{H}-\mathrm{Cosy}, \mathrm{HSQC}$ and $\mathrm{HMBC}$ were made. Melting point: the substance decomposed at $\mathrm{T}=166^{\circ} \mathrm{C}$; TLC (TBME: $\mathrm{MeOH}$ : ammonia 25\%/100:10:1): rf-value $=0.11$; IR: see spectrum in the supporting information; HR-MS (ESI, MeOH) calculated for $\mathrm{C}_{24} \mathrm{H}_{33} \mathrm{~N}_{5} \mathrm{O}_{1} \mathrm{Si}_{1}+\mathrm{H}^{+}: 436.25271 \mathrm{Da}$, found 436.25296 Da.

(R)-(6-(4-(Trimethylsilyl)-1H-1,2,3-triazol-1-yl)quinolin-4-yl)((1S,2S,4S,5R)-5-ethylquinuclidin-2yl)methanol (20): the title compound was synthesized from $11(0.5 \mathrm{~g}, 1.5 \mathrm{mmol})$ to afford 20 (0.57 g, yield: $89 \%)$ as a honey-type liquid. ${ }^{1} \mathrm{H}-\mathrm{NMR}\left(\mathrm{CDCl}_{3}, 300 \mathrm{MHz}\right) \delta 8.63(\mathrm{~d}, J=4.5 \mathrm{~Hz}$, H-2' $), 8.35$ (bs, H-Triazole-5), $8.24\left(\mathrm{~d}, J=1.9 \mathrm{~Hz}, \mathrm{H}-5^{\prime}\right), 8.00\left(\mathrm{~d}, J=9.1 \mathrm{~Hz}, \mathrm{H}-8^{\prime}\right), 7.95(\mathrm{dd}$, $\left.J=9.1 \mathrm{~Hz}, J=1.9 \mathrm{~Hz}, \mathrm{H}-7^{\prime}\right), 7.51\left(\mathrm{~d}, J=4.5 \mathrm{~Hz}, \mathrm{H}-3^{\prime}\right), 5.84-5.73(\mathrm{~m}, \mathrm{H}-9), 5.693 .73-3.57$ (m, H-6), 3.16-3.02 (m, H-2, H-8), 2.77-2.63 H-6), 2.47-2.33 (m, H-2), 1.93-1.77 (m; H-4, H-7), 1.57-1.40 (m, H-3, H-5, H-7), 1.29-1.15 (m, H-5, H-10, H-10), 0.78 (t, J = 7.3 Hz, H-11, $\mathrm{H}-11, \mathrm{H}-11)$, and $0.43\left(\mathrm{~s}, 9 \mathrm{H},-\mathrm{Si}\left(\mathrm{CH}_{3}\right)_{3}\right) ;{ }^{13} \mathrm{C}-\mathrm{NMR}\left(\mathrm{CDCl}_{3}, 75 \mathrm{MHz}\right) \delta 150.50\left(\mathrm{CH}, \mathrm{C}-2^{\prime}\right)$, $149.18\left(\mathrm{C}_{\mathrm{q}}, \mathrm{C}-4^{\prime}\right), 147.58\left(\mathrm{C}_{\mathrm{q}}, \mathrm{C}-\right.$ Triazole-4), $147.04\left(\mathrm{C}_{\mathrm{q}}, \mathrm{C}-8 \mathrm{a}^{\prime}\right) ; 134.45\left(\mathrm{C}_{\mathrm{q}}, \mathrm{C}-6^{\prime}\right), 131.89$ $\left(\mathrm{CH}, \mathrm{C}-8^{\prime}\right), 127.83\left(\mathrm{CH}, \mathrm{C}-\right.$ Triazole-5), $125.49\left(\mathrm{C}_{\mathrm{q}}, \mathrm{C}-4 \mathrm{a}^{\prime}\right), 121.64\left(\mathrm{CH}, \mathrm{C}-7^{\prime}\right), 119.53(\mathrm{CH}$, C-3'), $112.84\left(\mathrm{CH}, \mathrm{C}-5^{\prime}\right), 69.96(\mathrm{CH}, \mathrm{C}-9), 60.36(\mathrm{CH}, \mathrm{C}-8), 57.97\left(\mathrm{CH}_{2}, \mathrm{C}-2\right), 43.23\left(\mathrm{CH}_{2}\right.$, C-6), $36.95(\mathrm{CH}, \mathrm{C}-3), 27.39\left(\mathrm{CH}_{2}, \mathrm{C}-5\right), 27.37\left(\mathrm{CH}_{2}, \mathrm{C}-10\right), 25.18(\mathrm{CH}, \mathrm{C}-4), 20,76\left(\mathrm{CH}_{2}\right.$, $\mathrm{C}-7), 11.88\left(\mathrm{CH}_{3}, \mathrm{C}-11\right)$, and $-1.11\left(\mathrm{CH}_{3},-\mathrm{SiMe} 3\right) ; \mathrm{H}, \mathrm{H}-\mathrm{Cosy}, \mathrm{HSQC}$ and $\mathrm{HMBC}$ were made. Melting point: the substance decomposed at $\mathrm{T}=136{ }^{\circ} \mathrm{C}$; TLC (TBME: $\mathrm{MeOH}$ : ammonia 
25\%/100:10:1): rf-value $=0.16$; IR: see spectrum in the supporting information; HR-MS (ESI, MeOH) calculated for $\mathrm{C}_{24} \mathrm{H}_{33} \mathrm{~N}_{5} \mathrm{O}_{1} \mathrm{Si}_{1}+\mathrm{H}^{+}$: $436.25271 \mathrm{Da}$, found 436.25305 Da.

\subsubsection{Synthesis of the Triazole-Derivatives 15, 17, 19 and 21 via Desilylation}

General procedure: a purified Cinchona TMS-triazole (1.00 eq.) was suspended in a mixture $t \mathrm{Bu}-\mathrm{OH} / \mathrm{H}_{2} \mathrm{O}\left(1: 1\right.$ ratio) and $\mathrm{K}_{2} \mathrm{CO}_{3}$ (2.0 equiv) was added. The reaction mixture was rigorously stirred for $48 \mathrm{~h}$. Upon completion of the reaction, EtOAc was added and the organic layer was separated, washed with water, brine, dried over $\mathrm{Na}_{2} \mathrm{SO}_{4}$, filtered and concentrated in vacuo. The residues obtained were purified by flash column chromatography using MTBE: $\mathrm{MeOH}$ as a gradient elution to afford the corresponding triazole products.

(S)-(6-(1H-1,2,3-Triazol-1-yl)quinolin-4-yl)((1S,2R,4S,5R)-5-vinylquinuclidin-2-yl)methanol (15): the title compound was synthesized from $14(1.0 \mathrm{~g}, 2.3 \mathrm{mmol})$ to afford $15(672 \mathrm{mg}$, yield: $81 \%)$ as a white solid. ${ }^{1} \mathrm{H}-\mathrm{NMR}\left(\mathrm{CDCl}_{3}, 600 \mathrm{MHz}\right) \delta 8.68\left(\mathrm{~d}, J=4.5 \mathrm{~Hz}, \mathrm{H}-2^{\prime}\right), 8.34(\mathrm{~d}$, $\left.J=2.2 \mathrm{~Hz}, \mathrm{H}-5^{\prime}\right), 8.23\left(\mathrm{~d}, J=0.8 \mathrm{~Hz}, \mathrm{H}-\right.$ Triazole-5), $8.04\left(\mathrm{~d}, J=9.0 \mathrm{~Hz}, \mathrm{H}-8^{\prime}\right), 7.93(\mathrm{dd}$, $\left.J=9.0 \mathrm{~Hz}, J=2.2 \mathrm{~Hz}, \mathrm{H}-7^{\prime}\right), 7.77\left(\mathrm{~d}, J=0.8 \mathrm{~Hz}, \mathrm{H}-\right.$ Triazole-4), $7.58\left(\mathrm{~d}, J=4.5 \mathrm{~Hz}, \mathrm{H}-3^{\prime}\right), 6.04$ $(\mathrm{ddd}, J=16.7 \mathrm{~Hz}, J=10.3 \mathrm{~Hz}, J=7.5 \mathrm{~Hz}, \mathrm{H}-10), 5.69-5.66$ (m, H-9), 5.08-5.01 (m, H-11, H-11), 3.33-3.25 (m, H-2), 3.06-3.00 (m, H-8), 2.88-2.78 (m, H-2, H-6), 2.72-2.64 (m, H-6), 2.26-2.19 (m, H-3), 2.12-2.03 (m, H-7), 1.80-1.75 (m, H-4), 1.56-1.45 (m, H-5, H-5), and 1.28-1.20 (m, $\mathrm{H}-7) ;{ }^{13} \mathrm{C}-\mathrm{NMR}\left(\mathrm{CDCl}_{3}, 150 \mathrm{MHz}\right) \delta 150.71\left(\mathrm{CH}, \mathrm{C}-2^{\prime}\right), 150.24\left(\mathrm{C}_{\mathrm{q}}, \mathrm{C}-4^{\prime}\right), 147.13\left(\mathrm{C}_{\mathrm{q}}, \mathrm{C}-8 \mathrm{a}^{\prime}\right)$; $140.30(\mathrm{CH}, \mathrm{C}-10) ; 134.54\left(\mathrm{CH}, \mathrm{C}-\right.$ Triazole-4), $134.22\left(\mathrm{C}_{\mathrm{q}}, \mathrm{C}-6^{\prime}\right), 131.86\left(\mathrm{CH} ; \mathrm{C}-8^{\prime}\right), 125.78$ $\left(\mathrm{C}_{\mathrm{q}}, \mathrm{C}-4 \mathrm{a}^{\prime}\right), 122.07\left(\mathrm{CH}, \mathrm{C}-\right.$ Triazole-5), $121.41\left(\mathrm{CH}, \mathrm{C}-7^{\prime}\right), 119.60\left(\mathrm{CH} ; \mathrm{C}-3^{\prime}\right), 114.73\left(\mathrm{CH}_{2}\right.$, C-11), $113.70\left(\mathrm{CH}, \mathrm{C}-5^{\prime}\right), 71.11(\mathrm{CH}, \mathrm{C}-9), 60.25(\mathrm{CH}, \mathrm{C}-8), 49.73\left(\mathrm{CH}_{2}, \mathrm{C}-6\right), 49.11\left(\mathrm{CH}_{2}\right.$, $\mathrm{C}-2), 39.78(\mathrm{CH}, \mathrm{C}-3), 28.01(\mathrm{CH}, \mathrm{C}-4), 26.16\left(\mathrm{CH}_{2}, \mathrm{C}-5\right)$, and $21.42\left(\mathrm{CH}_{2}, \mathrm{C}-7\right) ; \mathrm{H}, \mathrm{H}-\mathrm{Cosy}$, HSQC and HMBC were made. Melting point: the substance decomposed at $\mathrm{T}=117^{\circ} \mathrm{C}$; TLC (TBME: $\mathrm{MeOH}$ : ammonia 25\%/100:10:1): rf-value $=0.56$; IR: see spectrum in the supporting information; HR-MS (ESI, MeOH) calculated for $\mathrm{C}_{21} \mathrm{H}_{23} \mathrm{~N}_{5} \mathrm{O}_{1}+\mathrm{H}^{+}: 362.19754$ $\mathrm{Da}$, found 362.19785 Da.

(R)-(6-(1H-1,2,3-Triazol-1-yl)quinolin-4-yl)((1S,2S,4S,5R)-5-vinylquinuclidin-2-yl)methanol (17): the title compound was synthesized from $16(1.0 \mathrm{~g}, 2.3 \mathrm{mmol})$ to afford $17(725 \mathrm{mg}$, yield: $87 \%)$ as a white solid. ${ }^{1} \mathrm{H}-\mathrm{NMR}\left(\mathrm{CDCl}_{3}, 600 \mathrm{MHz}\right) \delta 8.59\left(\mathrm{~d}, J=4.5 \mathrm{~Hz}, \mathrm{H}-2^{\prime}\right), 8.49$ (bs, H-Triazole-5), $8.37(\mathrm{~d}, J=1.8 \mathrm{~Hz}, \mathrm{H}-5), 8.00\left(\mathrm{dd}, J=9.1 \mathrm{~Hz}, J=1.8 \mathrm{~Hz}, \mathrm{H}-7^{\prime}\right), 7.95(\mathrm{~d}$, $\left.J=9.1 \mathrm{~Hz}, \mathrm{H}-8^{\prime}\right), 7.74$ (bs, H-Triazole-4), 7.59 (d, J = 4.5 Hz, H-3'), 5.77-5.68 (m, H-9), 5.70 $(\mathrm{ddd}, J=17.4 \mathrm{~Hz}, J=10.3 \mathrm{~Hz}, J=7.5 \mathrm{~Hz}, \mathrm{H}-10), 4.96-4.85$ (m, H-11, H-11), 3.67-3.53 (m, $\mathrm{H}-6), 3.12-2.98$ (m, H-2, H-8), 2.73-2.58 (m, H-2, H-6), 2.35-2.25 (m; H-3), 1.92-1.76 (m, $\mathrm{H}-4, \mathrm{H}-5, \mathrm{H}-7)$, and 1.60-1.48 (m, H-5, H-7); ${ }^{13} \mathrm{C}-\mathrm{NMR}\left(\mathrm{CDCl}_{3}, 150 \mathrm{MHz}\right) \delta 150.19(\mathrm{CH}$, C-2'), $150.09\left(\mathrm{C}_{\mathrm{q}}, \mathrm{C}-4^{\prime}\right), 146.62\left(\mathrm{C}_{\mathrm{q}}, \mathrm{C}-8 \mathrm{a}^{\prime}\right) ; 140.77(\mathrm{CH}, \mathrm{C}-10) ; 134.14(\mathrm{CH}, \mathrm{C}-$ Triazole-4), $134.12\left(\mathrm{C}_{\mathrm{q}}, \mathrm{C}-6^{\prime}\right), 131.31\left(\mathrm{CH} ; \mathrm{C}-8^{\prime}\right), 125.31\left(\mathrm{C}_{\mathrm{q}}, \mathrm{C}-4 \mathrm{a}^{\prime}\right), 122.07$ (CH, C-Triazole-5), 121.14 $\left(\mathrm{CH}, \mathrm{C}-7^{\prime}\right), 119.27\left(\mathrm{CH} ; \mathrm{C}-3^{\prime}\right), 114.41\left(\mathrm{CH}_{2}, \mathrm{C}-11\right), 112.73\left(\mathrm{CH}, \mathrm{C}-5^{\prime}\right), 70.03(\mathrm{CH}, \mathrm{C}-9), 60.22$ $(\mathrm{CH}, \mathrm{C}-8), 56.06\left(\mathrm{CH}_{2}, \mathrm{C}-2\right), 42.70\left(\mathrm{CH}_{2}, \mathrm{C}-6\right), 39.06(\mathrm{CH}, \mathrm{C}-3), 27.29(\mathrm{CH}, \mathrm{C}-4), 26.73\left(\mathrm{CH}_{2}\right.$, $\mathrm{C}-5)$, and $20.92\left(\mathrm{CH}_{2}, \mathrm{C}-7\right) ; \mathrm{H}, \mathrm{H}-\mathrm{Cosy}, \mathrm{HSQC}$ and $\mathrm{HMBC}$ were made. Melting point: the substance decomposed at $\mathrm{T}=92{ }^{\circ} \mathrm{C}$; TLC (TBME: $\mathrm{MeOH}$ : ammonia 25\%/100:10:1): rf-value $=0.63$; IR: see spectrum in the supporting information; HR-MS (ESI, MeOH) calculated for $\mathrm{C}_{21} \mathrm{H}_{23} \mathrm{~N}_{5} \mathrm{O}_{1}+\mathrm{H}^{+}$: 362.19754 Da, found 362.19796 Da.

(S)-(6-(1H-1,2,3-Triazol-1-yl)quinolin-4-yl)((1S,2R,4S,5R)-5-ethylquinuclidin-2-yl)methanol (19): the title compound was synthesized from $18(0.5 \mathrm{~g}, 1.15 \mathrm{mmol})$ to afford 19 ( $359 \mathrm{mg}$, yield: $86 \%)$ as an off-white solid. ${ }^{1} \mathrm{H}-\mathrm{NMR}\left(\mathrm{CDCl}_{3}, 300 \mathrm{MHz}\right) \delta 8.54\left(\mathrm{~d}, \mathrm{~J}=4.5 \mathrm{~Hz}, \mathrm{H}-2^{\prime}\right), 8.26$ $\left(\mathrm{d}, J=2.2 \mathrm{~Hz}, \mathrm{H}-5^{\prime}\right), 8.35\left(\mathrm{~d}, J=0.8 \mathrm{~Hz}, \mathrm{H}-\right.$ Triazole-5), $8.98\left(\mathrm{~d}, J=9.0 \mathrm{~Hz}, \mathrm{H}-8^{\prime}\right), 7.65(\mathrm{dd}$, $\left.J=9.0 \mathrm{~Hz}, J=2.2 \mathrm{~Hz}, \mathrm{H}-7^{\prime}\right), 7.98\left(\mathrm{~d}, J=0.8 \mathrm{~Hz}, \mathrm{H}-\right.$ Triazole-4), $7.94\left(\mathrm{~d}, J=4.5 \mathrm{~Hz}, \mathrm{H}-3^{\prime}\right)$, 5.87-5.77 (m, H-9), 3.31-3.20 (m, H-2), 3.11-3.08 (m, H-8), 2.85-2.73 (m, H-2, H-6), 2.69-2.58 (m, H-6), 2.21-2.15 (m, H-3), 2.32-2.23 (m, H-7), 1.85-1.80 (m, H-4), 1.51-1.40 (m, H-5, H-5, $\mathrm{H}-10, \mathrm{H}-10), 1.22-1.14(\mathrm{~m}, \mathrm{H}-7)$, and $0.81(\mathrm{t}, J=7.2 \mathrm{~Hz}, \mathrm{H}-11, \mathrm{H}-11, \mathrm{H}-11) ;{ }^{13} \mathrm{C}-\mathrm{NMR}\left(\mathrm{CDCl}_{3}\right.$, $75 \mathrm{MHz}) \delta{ }^{13} \mathrm{C}-\mathrm{NMR}\left(\mathrm{CDCl}_{3}, 75 \mathrm{MHz}\right) \delta 150.81\left(\mathrm{CH}, \mathrm{C}-2^{\prime}\right), 149.80\left(\mathrm{C}_{\mathrm{q}}, \mathrm{C}-4^{\prime}\right), 147.27\left(\mathrm{C}_{\mathrm{q}}\right.$, 
C-8a'), $134.62\left(\mathrm{CH}, \mathrm{H}-\right.$ Triazole-4), $134.30\left(\mathrm{C}_{\mathrm{q}}, \mathrm{C}-6^{\prime}\right), 132.07\left(\mathrm{CH} ; \mathrm{C}-8^{\prime}\right), 125.75\left(\mathrm{C}_{\mathrm{q}}, \mathrm{C}-4 \mathrm{a}^{\prime}\right)$, $122.12\left(\mathrm{CH}, \mathrm{H}-\right.$ Triazole-5), $121.40\left(\mathrm{CH}, \mathrm{C}-7^{\prime}\right), 119.60\left(\mathrm{CH} ; \mathrm{C}-3^{\prime}\right), 113.56\left(\mathrm{CH}, \mathrm{C}-5^{\prime}\right), 70.93(\mathrm{CH}$, C-9), $60.38(\mathrm{CH}, \mathrm{C}-8), 50.74\left(\mathrm{CH}_{2}, \mathrm{C}-6\right), 49.87\left(\mathrm{CH}_{2}, \mathrm{C}-2\right), 37.12(\mathrm{CH}, \mathrm{C}-3), 26.76(\mathrm{CH}, \mathrm{C}-4)$, $26.02\left(\mathrm{CH}_{2}, \mathrm{C}-10\right), 25.08\left(\mathrm{CH}_{2}, \mathrm{C}-5\right), 21.09\left(\mathrm{CH}_{2}, \mathrm{C}-7\right)$, and $11.92\left(\mathrm{CH}_{3}, \mathrm{C}-11\right) ; \mathrm{H}, \mathrm{H}-\mathrm{Cosy}$, HSQC and HMBC were made. Melting point: Substance decompose at $\mathrm{T}=143{ }^{\circ} \mathrm{C}$; TLC (TBME: MeOH: ammonia 25\%/100:10:1): rf-value =0.45; IR: see spectrum in the supporting information; HR-MS (ESI, MeOH) calculated for $\mathrm{C}_{21} \mathrm{H}_{25} \mathrm{~N}_{5} \mathrm{O}_{1}+\mathrm{H}^{+}: 364.21319 \mathrm{Da}$, found 364.21347 Da.

(R)-(6-(1H-1,2,3-Triazol-1-yl)quinolin-4-yl)((1S,2S,4S,5R)-5-ethylquinuclidin-2-yl)methanol (21): the title compound was synthesized from $20(0.5 \mathrm{~g}, 1.15 \mathrm{mmol})$ to afford $21(346 \mathrm{mg}$, yield: $84 \%)$ as an off-white solid. ${ }^{1} \mathrm{H}-\mathrm{NMR}\left(\mathrm{CDCl}_{3}, 300 \mathrm{MHz}\right) \delta 8.68\left(\mathrm{~d}, J=4.5 \mathrm{~Hz}, \mathrm{H}-2^{\prime}\right), 8.37(\mathrm{~d}$, $J=2.3 \mathrm{~Hz}, \mathrm{H}-5), 8.29\left(\mathrm{~d}, J=1.0 \mathrm{~Hz}, \mathrm{H}-\right.$ Triazole-5), $8.05\left(\mathrm{~d}, J=9.1 \mathrm{~Hz}, \mathrm{H}-8^{\prime}\right), 7.96(\mathrm{dd}$, $\left.J=9.1 \mathrm{~Hz}, J=2.3 \mathrm{~Hz}, \mathrm{H}-7^{\prime}\right), 7.80\left(\mathrm{~d}, J=1.0 \mathrm{~Hz}, \mathrm{H}-\right.$ Triazole-4), $7.57\left(\mathrm{~d}, J=4.57 \mathrm{~Hz}, \mathrm{H}-3^{\prime}\right)$, 5.69-5.59 (m, H-9), 3.47-3.41 (m, H-6), 3.11-2.96 (m, H-2, H-8), 2.65-2.55 (m, H-6), 2.35-2.29 (m, H-2), 1.79-1.71 (m; H-4, H-5, H-7), 1.60-1.41 (m, H-3, H-10, H-10), 1.34-1.18 (m, H-5, $\mathrm{H}-7)$, and $0.79(\mathrm{t}, J=7.3 \mathrm{~Hz}, \mathrm{H}-11, \mathrm{H}-11, \mathrm{H}-11) ;{ }^{13} \mathrm{C}-\mathrm{NMR}\left(\mathrm{CDCl}_{3}, 75 \mathrm{MHz}\right) \delta 150.79(\mathrm{CH}$, $\left.\mathrm{C}-2^{\prime}\right), 150.07\left(\mathrm{C}_{\mathrm{q}}, \mathrm{C}-4^{\prime}\right), 147.25\left(\mathrm{C}_{\mathrm{q}}, \mathrm{C}-8 \mathrm{a}^{\prime}\right)$; $134.60\left(\mathrm{CH}, \mathrm{H}-\right.$ Triazole-4), $134.36\left(\mathrm{C}_{\mathrm{q}}, \mathrm{C}-6^{\prime}\right)$, $132.01\left(\mathrm{CH}, \mathrm{C}-8^{\prime}\right), 125.80\left(\mathrm{C}_{\mathrm{q}}, \mathrm{C}-4 \mathrm{a}^{\prime}\right), 122.27\left(\mathrm{CH}, \mathrm{H}-\right.$ Triazole-5), $121.49\left(\mathrm{CH}, \mathrm{C}-7^{\prime}\right), 119.65$ $\left(\mathrm{CH}, \mathrm{C}-3^{\prime}\right), 113.51\left(\mathrm{CH}, \mathrm{C}-5^{\prime}\right), 70.85(\mathrm{CH}, \mathrm{C}-9), 60.39(\mathrm{CH}, \mathrm{C}-8), 58.18\left(\mathrm{CH}_{2}, \mathrm{C}-2\right), 43.03$ $\left(\mathrm{CH}_{2}, \mathrm{C}-6\right), 37.20(\mathrm{CH}, \mathrm{C}-3), 27.88\left(\mathrm{CH}_{2}, \mathrm{C}-5\right), 27.52\left(\mathrm{CH}_{2}, \mathrm{C}-10\right), 25.27(\mathrm{CH}, \mathrm{C}-4), 21.70$ $\left(\mathrm{CH}_{2}, \mathrm{C}-7\right)$, and $11.96\left(\mathrm{CH}_{3}, \mathrm{C}-11\right) ; \mathrm{H}, \mathrm{H}-\mathrm{Cosy}, \mathrm{HSQC}$ and $\mathrm{HMBC}$ were made. Melting point: the substance decomposed at $\mathrm{T}=106{ }^{\circ} \mathrm{C}$; TLC (TBME: MeOH: ammonia 25\%/100:10:1): rf-value $=0.58 ;$ IR: see spectrum in the supporting information; HR-MS (ESI, MeOH) calculated for $\mathrm{C}_{21} \mathrm{H}_{25} \mathrm{~N}_{5} \mathrm{O}_{1}+\mathrm{H}^{+}: 364.21319 \mathrm{Da}$, found $364.21358 \mathrm{Da}$.

\subsubsection{Synthesis of the Quinuclidine Containing Triazole-Derivatives 24-31}

General procedure: a dropwise a solution of $\mathrm{NaNO}_{2}(1.2 \mathrm{eq})$ in $\mathrm{H}_{2} \mathrm{O}(0.33 \mathrm{~g} / \mathrm{mL})$ was added to a mixture of Cinchona amine $(1.0 \mathrm{eq})$ and $15 \% \mathrm{HCl}(0.1 \mathrm{~g}$ amine $/ \mathrm{mL})$ cooled at $-5{ }^{\circ} \mathrm{C}$. After the completion of the addition, the reaction mixture was stirred at this temperature for $60 \mathrm{~min}$. A solution of $\mathrm{NaN}_{3}(1.7 \mathrm{eq})$ in $\mathrm{H}_{2} \mathrm{O}(0.2 \mathrm{~g} / \mathrm{mL})$ was added dropwise to the reaction mixture at $0{ }^{\circ} \mathrm{C}$. After the addition was finished, the reaction mixture was maintained at $0{ }^{\circ} \mathrm{C}$ for $2 \mathrm{~h}$ and then stirred at room temperature for $12 \mathrm{~h}$. The product was precipitated by the addition of $\mathrm{NaOH}(20 \%)$ until $\mathrm{pH} 10$. Upon filtration, the solid was washed with distilled water and dried. Crystallization from DCM presented the Cinchona azides as yellow solids in good yields (70-90\%).

(S)-(6-(4-((1S,3R,4S,6R)-6-(Hydroxymethyl)quinuclidin-3-yl)-1H-1,2,3-triazol-1-yl)quinolin-4$y l)((1 S, 2 R, 4 S, 5 R)-5$-vinylquinuclidin-2-yl)methanol (24): the title compound was synthesized from $8(1.0 \mathrm{~g}, 3.0 \mathrm{mmol})$ to afford $24(925 \mathrm{mg}$, yield: $62 \%)$ as a reddish solid. ${ }^{1} \mathrm{H}-\mathrm{NMR}$ $\left(\mathrm{CDCl}_{3}, 400 \mathrm{MHz}\right) \delta 8.87-8.72(1 \mathrm{H}), 8.41-8.28(2 \mathrm{H}), 8.39-7.54(2 \mathrm{H}), 7.69-7.58(1 \mathrm{H}), 6.27-$ $6.56(1 \mathrm{H}), 5.73-5.65(1 \mathrm{H}), 5.21-5.11(2 \mathrm{H}), 3.75-3.29(4 \mathrm{H}), 3.11-2.75(6 \mathrm{H}), 2.89-2.75(2 \mathrm{H})$, 2.46-2.33 (1 H), 2.11-2.01 (2 H), 1.78-1.35 (7 H), 1.29-1.15 (1 H), and 0.89-0.76 $(1 \mathrm{H}) ;{ }^{13} \mathrm{C}$ $\operatorname{NMR}\left(\mathrm{CDCl}_{3}, 100 \mathrm{MHz}\right) \delta 151.20\left(\mathrm{C}_{\mathrm{q}}\right), 150.54(\mathrm{CH}), 150.34\left(\mathrm{C}_{\mathrm{q}}\right), 146.96\left(\mathrm{C}_{\mathrm{q}}\right) ; 140.45(\mathrm{CH})$, $134.55(\mathrm{CH}), 131.79(\mathrm{CH}), 125.49\left(\mathrm{C}_{\mathrm{q}}\right), 121.19(\mathrm{CH}), 119.36(\mathrm{CH}), 119.22(\mathrm{CH}), 114.73\left(\mathrm{CH}_{2}\right)$, $112.50(\mathrm{CH}), 70.67(\mathrm{CH}), 62.64\left(\mathrm{CH}_{2}\right), 59.94(\mathrm{CH}), 57.30(\mathrm{CH}), 49.83\left(\mathrm{CH}_{2}\right), 49.30\left(\mathrm{CH}_{2}\right), 48.89$ $\left(\mathrm{CH}_{2}\right), 49.77\left(\mathrm{CH}_{2}\right), 39.98(\mathrm{CH}), 33.36(\mathrm{CH}), 28.15(\mathrm{CH}), 27.55\left(\mathrm{CH}_{2}\right), 26.35(\mathrm{CH}), 26.16\left(\mathrm{CH}_{2}\right)$, $23.71\left(\mathrm{CH}_{2}\right)$, and $20.08\left(\mathrm{CH}_{2}\right)$; Melting point: the substance decomposed at $\mathrm{T}=126{ }^{\circ} \mathrm{C}$; TLC (TBME: MeOH: ammonia 25\%/100:10:1): rf-value =0.21; IR: see spectrum in the supporting information; HR-MS (ESI, $\mathrm{MeOH}$ ) calculated for $\mathrm{C}_{29} \mathrm{H}_{36} \mathrm{~N}_{6} \mathrm{O}_{2}+\mathrm{H}^{+}: 501.29725 \mathrm{Da}$, found 501.29739 Da.

(S)-(6-(4-((1S,3R,4S,6S)-6-(Hydroxymethyl)quinuclidin-3-yl)-1H-1,2,3-triazol-1-yl)quinolin-4$y l)((1 S, 2 R, 4 S, 5 R)-5$-vinylquinuclidin-2-yl)methanol (25): the title compound was synthesized from $8(1.0 \mathrm{~g}, 3.0 \mathrm{mmol})$ to afford $25(1.0 \mathrm{~g}$, yield: $68 \%)$ as a pink solid. ${ }^{1} \mathrm{H}-\mathrm{NMR}$ $\left(\mathrm{CDCl}_{3}, 400 \mathrm{MHz}\right) \delta 8.71-8.64(1 \mathrm{H}), 8.37-8.25(2 \mathrm{H}), 8.00-7.88(2 \mathrm{H}), 7.62-7.56(1 \mathrm{H})$, 
6.16-6.04 (1 H), 5.82-5.70 (1 H), 5.13-5.01 (2 H), 3.64-3.19 (4 H), 3.16-2.82 (6 H), 2.80-2.69 $(2 \mathrm{H}), 2.32-2.21(1 \mathrm{H}), 2.19-2.08(2 \mathrm{H}), 1.82-1.43(7 \mathrm{H}), 1.25-1.13(1 \mathrm{H})$, and $0.94-0.83(1 \mathrm{H})$; ${ }^{13} \mathrm{C}-\mathrm{NMR}\left(\mathrm{CDCl}_{3}, 100 \mathrm{MHz}\right) \delta 151.73\left(\mathrm{C}_{\mathrm{q}}\right), 150.36(\mathrm{CH}), 150.24\left(\mathrm{C}_{\mathrm{q}}\right), 146.82\left(\mathrm{C}_{\mathrm{q}}\right) ; 140.17$ $(\mathrm{CH}), 134.35(\mathrm{CH}), 131.42(\mathrm{CH}), 125.60\left(\mathrm{C}_{\mathrm{q}}\right), 121.08(\mathrm{CH}), 119.35(\mathrm{CH}), 119.12(\mathrm{CH}), 114.71$ $\left(\mathrm{CH}_{2}\right), 112.86(\mathrm{CH}), 70.3(\mathrm{CH}), 63.33\left(\mathrm{CH}_{2}\right), 59.59(\mathrm{CH}), 57.28(\mathrm{CH}), 54.75\left(\mathrm{CH}_{2}\right), 49.61$ $\left(\mathrm{CH}_{2}\right), 49.20\left(\mathrm{CH}_{2}\right), 40.44\left(\mathrm{CH}_{2}\right), 39.75(\mathrm{CH}), 33.02(\mathrm{CH}), 27.96(\mathrm{CH}), 27.20\left(\mathrm{CH}_{2}\right), 27.06$ $(\mathrm{CH}), 25.97\left(\mathrm{CH}_{2}\right), 24.89\left(\mathrm{CH}_{2}\right)$, and $20.92\left(\mathrm{CH}_{2}\right)$. Melting point: the substance decomposed at $\mathrm{T}=119{ }^{\circ} \mathrm{C}$; TLC (TBME: MeOH: ammonia 25\%/100:10:1): rf-value $=0.20$; IR: see spectrum in the supporting information; HR-MS (ESI, MeOH) calculated for $\mathrm{C}_{29} \mathrm{H}_{36} \mathrm{~N}_{6} \mathrm{O}_{2}+\mathrm{H}^{+}$: 501.29725 Da, found 501.29742 Da.

(R)-(6-(4-((1S,3R,4S,6R)-6-(Hydroxymethyl)quinuclidin-3-yl)-1H-1,2,3-triazol-1-yl)quinolin4-yl)((1S,2S,4S,5R)-5-vinylquinuclidin-2-yl)methanol (26): the title compound was synthesized from $9(1.0 \mathrm{~g}, 3.0 \mathrm{mmol})$ to afford $26(1.1 \mathrm{~g}$, yield: $74 \%)$ as a light red solid. ${ }^{1} \mathrm{H}-\mathrm{NMR}$ $\left(\mathrm{CDCl}_{3}, 300 \mathrm{MHz}\right) \delta 8.61-8.54(1 \mathrm{H}), 8.67-8.54(2 \mathrm{H}), 8.13-7.87(2 \mathrm{H}), 7.68-7.46(1 \mathrm{H})$, 6.96-6.64 (1 H), 5.34-5.23 (1 H), 5.17-5.03 (2 H), 3.78-3.35 (4 H), 3.26-2.92 (6 H), 2.63-2.46 (2 H), 2.39-2.26 (1 H), 2.12-2.01 (2 H), 1.76-1.32 (7 H), 1.19-1.07 (1 H), and 0.97-0.85 (1 H); ${ }^{13} \mathrm{C}-\mathrm{NMR}\left(\mathrm{CDCl}_{3}, 75 \mathrm{MHz}\right) \delta 152.08\left(\mathrm{C}_{\mathrm{q}}\right), 150.67(\mathrm{CH}), 148.43\left(\mathrm{C}_{\mathrm{q}}\right), 147.67\left(\mathrm{C}_{\mathrm{q}}\right) ; 141.21$ $(\mathrm{CH}), 133.96(\mathrm{CH}), 132.54(\mathrm{CH}), 126.31\left(\mathrm{C}_{\mathrm{q}}\right), 122.45(\mathrm{CH}), 120.08(\mathrm{CH}), 119.23(\mathrm{CH}), 115.76$ $\left(\mathrm{CH}_{2}\right), 111.47(\mathrm{CH}), 69.93(\mathrm{CH}), 62.48\left(\mathrm{CH}_{2}\right), 61.13\left(\mathrm{CH}_{2}\right), 60.67(\mathrm{CH}), 57.79\left(\mathrm{CH}_{2}\right), 56.36$ $\left(\mathrm{CH}_{2}\right), 54.76\left(\mathrm{CH}_{2}\right), 42.98\left(\mathrm{CH}_{2}\right), 40.54(\mathrm{CH}), 34.65(\mathrm{CH}), 27.87(\mathrm{CH}), 26.03(\mathrm{CH}), 25.53\left(\mathrm{CH}_{2}\right)$, $24.52\left(\mathrm{CH}_{2}\right), 21.73(\mathrm{CH})$, and $19.26\left(\mathrm{CH}_{2}\right)$. Melting point: the substance decomposed at $\mathrm{T}=110^{\circ} \mathrm{C}$; TLC (TBME: MeOH: ammonia 25\%/100:10:1): rf-value =0.16; IR: see spectrum in the supporting information; HR-MS (ESI, MeOH) calculated for $\mathrm{C}_{29} \mathrm{H}_{36} \mathrm{~N}_{6} \mathrm{O}_{2}+\mathrm{H}^{+}$: 501.29725 Da, found 501.29747 Da.

(R)-(6-(4-((1S,3R,4S,6S)-6-(Hydroxymethyl)quinuclidin-3-yl)-1H-1,2,3-triazol-1-yl)quinolin-4$y l)((1 S, 2 S, 4 S, 5 R)-5-v i n y l q u i n u c l i d i n-2-y l) m e t h a n o l(27)$ : the title compound was synthesized from $9(1.0 \mathrm{~g}, 3.0 \mathrm{mmol})$ to afford $27(1.15 \mathrm{mg}$, yield: $76 \%)$ as a pink solid. ${ }^{1} \mathrm{H}-\mathrm{NMR}\left(\mathrm{CDCl}_{3}\right.$, $300 \mathrm{MHz}) \delta 8.71-8.64(1 \mathrm{H}), 8.37-8.25(2 \mathrm{H}), 8.00-7.88(2 \mathrm{H}), 7.62-7.56(1 \mathrm{H}), 6.16-6.04(1 \mathrm{H})$, 5.82-5.70 (1 H), 5.13-5.01 (2 H), 3.64-3.19 (4 H), 3.16-2.82 (6 H), 2.80-2.69 (2 H), 2.32-2.21 $(1 \mathrm{H}), 2.19-2.08(2 \mathrm{H}), 1.82-1.43(7 \mathrm{H}), 1.25-1.13(1 \mathrm{H})$, and $0.94-0.83(1 \mathrm{H}) ;{ }^{13} \mathrm{C}-\mathrm{NMR}\left(\mathrm{CDCl}_{3}\right.$, $75 \mathrm{MHz}) \delta 151.99\left(\mathrm{C}_{\mathrm{q}}\right), 150.65(\mathrm{CH}), 147.30\left(\mathrm{C}_{\mathrm{q}}\right), 147.26\left(\mathrm{C}_{\mathrm{q}}\right) ; 140.48(\mathrm{CH}), 133.16(\mathrm{CH})$, $132.17(\mathrm{CH}), 125.47\left(\mathrm{C}_{\mathrm{q}}\right), 121.58(\mathrm{CH}), 119.79(\mathrm{CH}), 119.33(\mathrm{CH}), 115.07\left(\mathrm{CH}_{2}\right), 112.09(\mathrm{CH})$, $69.33(\mathrm{CH}), 63.43\left(\mathrm{CH}_{2}\right), 60.54\left(\mathrm{CH}_{2}\right), 60.34(\mathrm{CH}), 57.43\left(\mathrm{CH}_{2}\right), 55.89\left(\mathrm{CH}_{2}\right), 54.16\left(\mathrm{CH}_{2}\right)$, $43.25\left(\mathrm{CH}_{2}\right), 40.19(\mathrm{CH}), 33.11(\mathrm{CH}), 27.49(\mathrm{CH}), 26.96(\mathrm{CH}), 26.79\left(\mathrm{CH}_{2}\right), 24.71\left(\mathrm{CH}_{2}\right)$, $21.00(\mathrm{CH})$, and $19.88\left(\mathrm{CH}_{2}\right)$. Melting point: the substance decomposed at $\mathrm{T}=103{ }^{\circ} \mathrm{C}$; TLC (TBME: MeOH: ammonia 25\%/100:10:1): rf-value =0.12; IR: see spectrum in the supporting information; HR-MS (ESI, $\mathrm{MeOH}$ ) calculated for $\mathrm{C}_{29} \mathrm{H}_{36} \mathrm{~N}_{6} \mathrm{O}_{2}+\mathrm{H}^{+}$: $501.29725 \mathrm{Da}$, found 501.29753 Da.

(S)-(6-(4-((1S,3R,4S,6R)-6-(Hydroxymethyl)quinuclidin-3-yl)-1H-1,2,3-triazol-1-yl)quinolin-4yl)((1S,2R,4S,5R)-5-ethylquinuclidin-2-yl)methanol (28): the title compound was synthesized from $10(0.5 \mathrm{~g}, 1.5 \mathrm{mmol})$ to afford $28(490 \mathrm{mg}$, yield: $65 \%)$ as an off-white solid. ${ }^{1} \mathrm{H}-\mathrm{NMR}$ $\left(\mathrm{CDCl}_{3}, 300 \mathrm{MHz}\right) \delta 8.75-8.54(1 \mathrm{H}), 8.28-8.14(2 \mathrm{H}), 8.01-7.82(2 \mathrm{H}), 7.59-7.54(1 \mathrm{H})$, 5.73-5.61 (1 H), 3.79-3.27 (4 H), 3.21-2.88 (6 H), 2.81-2.61 (2 H), 2.41-2.32 (1 H), 2.26-2.18 (2 H), 1.89-1.31 (9 H), 1.21-1.12 (1 H), and 0.99-0.81 $(4 \mathrm{H}) ;{ }^{13} \mathrm{C}-\mathrm{NMR}\left(\mathrm{CDCl}_{3}, 75 \mathrm{MHz}\right) \delta$ $152.71\left(\mathrm{C}_{\mathrm{q}}\right), 150.98(\mathrm{CH}), 150.37\left(\mathrm{C}_{\mathrm{q}}\right), 147.63\left(\mathrm{C}_{\mathrm{q}}\right), 135.01(\mathrm{CH}), 132.23(\mathrm{CH}), 126.29\left(\mathrm{C}_{\mathrm{q}}\right)$, $121.78(\mathrm{CH}), 119.73(\mathrm{CH}), 118.61(\mathrm{CH}), 113.93(\mathrm{CH}), 71.35(\mathrm{CH}), 62.67\left(\mathrm{CH}_{2}\right), 60.59(\mathrm{CH})$, $58.79(\mathrm{CH}), 55.23\left(\mathrm{CH}_{2}\right), 50.69\left(\mathrm{CH}_{2}\right), 49.13\left(\mathrm{CH}_{2}\right), 41.65\left(\mathrm{CH}_{2}\right), 40.72(\mathrm{CH}), 33.19(\mathrm{CH})$, $28.17(\mathrm{CH}), 27.64\left(\mathrm{CH}_{2}\right), 27.13(\mathrm{CH}), 26.27\left(\mathrm{CH}_{2}\right), 25.93\left(\mathrm{CH}_{2}\right), 24.63\left(\mathrm{CH}_{2}\right), 21.47\left(\mathrm{CH}_{2}\right)$, and $12.65\left(\mathrm{CH}_{3}\right)$. Melting point: the substance decomposed at T $=145^{\circ} \mathrm{C}$; TLC (TBME: MeOH: ammonia 25\%/100:10:1): rf-value $=0.19$; IR: see spectrum in the supporting information; HR-MS (ESI, MeOH) calculated for $\mathrm{C}_{29} \mathrm{H}_{38} \mathrm{~N}_{6} \mathrm{O}_{2}+\mathrm{H}^{+}$: $503.31290 \mathrm{Da}$, found 503.31304 Da.

(S)-(6-(4-((1S,3R,4S,6S)-6-(Hydroxymethyl)quinuclidin-3-yl)-1H-1,2,3-triazol-1-yl)quinolin-4$y l)((1 S, 2 R, 4 S, 5 R)-5$-ethylquinuclidin-2-yl)methanol (29): the title compound was synthesized 
from $10(0.5 \mathrm{~g}, 1.5 \mathrm{mmol})$ to afford $29(580 \mathrm{mg}$, yield: $78 \%)$ as an off-white solid; ${ }^{1} \mathrm{H}-\mathrm{NMR}$ $\left(\mathrm{CDCl}_{3}, 400 \mathrm{MHz}\right) \delta$ 9.03-8.73 (1 H), 8.41-8.29 (2 H), 8.13-7.91 (2 H), 7.68-7.59 (1 H), 6.23-5.81 (1 H), 3.69-3.23 (4 H), 3.17-2.81 (6 H), 2.75-2.34 (2 H), 2.32-2.23 (1 H), 2.16-2.01 $(2 \mathrm{H}), 1.87-1.34(9 \mathrm{H}), 1.27-1.14(1 \mathrm{H})$, and $0.91-0.87(4 \mathrm{H}) ;{ }^{13} \mathrm{C}-\mathrm{NMR}\left(\mathrm{CDCl}_{3}, 100 \mathrm{MHz}\right)$ $\delta 151.54\left(\mathrm{C}_{\mathrm{q}}\right), 151.12(\mathrm{CH}), 150.27\left(\mathrm{C}_{\mathrm{q}}\right), 146.12\left(\mathrm{C}_{\mathrm{q}}\right), 135.69(\mathrm{CH}), 130.19(\mathrm{CH}), 125.94\left(\mathrm{C}_{\mathrm{q}}\right)$, $121.98(\mathrm{CH}), 120.15(\mathrm{CH}), 119.72(\mathrm{CH}), 113.26(\mathrm{CH}), 71.3(\mathrm{CH}), 63.99\left(\mathrm{CH}_{2}\right), 60.57(\mathrm{CH})$, $57.52(\mathrm{CH}), 55.75\left(\mathrm{CH}_{2}\right), 49.69\left(\mathrm{CH}_{2}\right), 49.13\left(\mathrm{CH}_{2}\right), 41.64\left(\mathrm{CH}_{2}\right), 40.73(\mathrm{CH}), 33.92(\mathrm{CH})$, 27.56 (CH), 27.21 $\left(\mathrm{CH}_{2}\right), 27.16(\mathrm{CH}), 25.47\left(\mathrm{CH}_{2}\right), 25.22\left(\mathrm{CH}_{2}\right), 24.83\left(\mathrm{CH}_{2}\right), 21.62\left(\mathrm{CH}_{2}\right)$, and $12.35\left(\mathrm{CH}_{3}\right)$. Melting point: the substance decomposed at $\mathrm{T}=138^{\circ} \mathrm{C}$; TLC (TBME: $\mathrm{MeOH}$ : ammonia $25 \% / 100: 10: 1)$ : rf-value $=0.21$; IR: see spectrum in the supporting information; HR-MS (ESI, MeOH) calculated for $\mathrm{C}_{29} \mathrm{H}_{38} \mathrm{~N}_{6} \mathrm{O}_{2}+\mathrm{H}^{+}: 503.31290 \mathrm{Da}$, found $503.31298 \mathrm{Da}$.

(R)-(6-(4-((1S,3R,4S,6R)-6-(Hydroxymethyl)quinuclidin-3-yl)-1H-1,2,3-triazol-1-yl)quinolin4-yl)((1S,2S,4S,5R)-5-ethylquinuclidin-2-yl)methanol (30): the title compound was synthesized from $11(0.5 \mathrm{~g}, 1.5 \mathrm{mmol})$ to afford $30(535 \mathrm{mg}$, yield: $72 \%)$ as an off-white solid. ${ }^{1} \mathrm{H}-\mathrm{NMR}\left(\mathrm{CDCl}_{3}, 300 \mathrm{MHz}\right) \delta 8.62-8.49(1 \mathrm{H}), 8.38-8.27(2 \mathrm{H}), 7.99-7.81(2 \mathrm{H}), 7.71-7.60$ $(1 \mathrm{H}), 5.89-5.65(1 \mathrm{H}), 5.17-4.89(2 \mathrm{H}), 3.73-3.29(4 \mathrm{H}), 3.21-2.94(6 \mathrm{H}), 2.81-2.73(2 \mathrm{H})$, 2.54-2.36 $(1 \mathrm{H}), 2.28-2.17(2 \mathrm{H}), 1.93-1.49(9 \mathrm{H}), 1.29-1.14(1 \mathrm{H})$, and $0.97-0.73(4 \mathrm{H}) ;{ }^{13} \mathrm{C}-$ NMR $\left(\mathrm{CDCl}_{3}, 75 \mathrm{MHz}\right) \delta 152.21\left(\mathrm{C}_{\mathrm{q}}\right), 151.25(\mathrm{CH}), 147.91\left(\mathrm{C}_{\mathrm{q}}\right), 146.66\left(\mathrm{C}_{\mathrm{q}}\right) ; 133.73(\mathrm{CH})$, $131.17(\mathrm{CH}), 125.97\left(\mathrm{C}_{\mathrm{q}}\right), 122.68(\mathrm{CH}), 120.19(\mathrm{CH}), 119.13(\mathrm{CH}), 112.34(\mathrm{CH}), 69.78(\mathrm{CH})$, $64.41\left(\mathrm{CH}_{2}\right), 61.64\left(\mathrm{CH}_{2}\right), 60.52(\mathrm{CH}), 58.67\left(\mathrm{CH}_{2}\right), 56.37\left(\mathrm{CH}_{2}\right), 54.73\left(\mathrm{CH}_{2}\right), 44.87\left(\mathrm{CH}_{2}\right)$, $40.36(\mathrm{CH}), 33.57(\mathrm{CH}), 28.19(\mathrm{CH}), 27.91(\mathrm{CH}), 26.71\left(\mathrm{CH}_{2}\right), 25.52\left(\mathrm{CH}_{2}\right), 24.11\left(\mathrm{CH}_{2}\right)$, $21.68(\mathrm{CH}), 20.18\left(\mathrm{CH}_{2}\right)$, and $12.37\left(\mathrm{CH}_{3}\right)$. Melting point: the substance decomposed at $\mathrm{T}=132{ }^{\circ} \mathrm{C}$; TLC (TBME: $\mathrm{MeOH}$ : ammonia 25\%/100:10:1): rf-value = 0.14; IR: see spectrum in the supporting information; HR-MS (ESI, $\mathrm{MeOH})$ calculated for $\mathrm{C}_{29} \mathrm{H}_{38} \mathrm{~N}_{6} \mathrm{O}_{2}+\mathrm{H}^{+}$: 503.31290 Da, found 503.31312 Da.

(R)-(6-(4-((1S,3R,4S,6S)-6-(Hydroxymethyl)quinuclidin-3-yl)-1H-1,2,3-triazol-1-yl)quinolin-4$y l)((1 S, 2 S, 4 S, 5 R)-5$-ethylquinuclidin-2-yl)methanol (31): the title compound was synthesized from $11(0.5 \mathrm{~g}, 1.5 \mathrm{mmol})$ to afford $31(610 \mathrm{mg}$, yield: $82 \%)$ as an off-white solid. ${ }^{1} \mathrm{H}-$ $\operatorname{NMR}\left(\mathrm{CDCl}_{3}, 300 \mathrm{MHz}\right) \delta 8.89-8.71(1 \mathrm{H}), 8.39-8.31(2 \mathrm{H}), 8.23-8.12(2 \mathrm{H}), 7.73-7.66(1 \mathrm{H})$, 5.79-5.65 (1 H), 5.56-5.23 (2 H), 3.81-3.39 (4 H), 3.25-2.93 (6 H), 2.85-2.71 (2 H), 2.54-2.35 $(1 \mathrm{H}), 2.23-2.13(2 \mathrm{H}), 1.97-1.51(9 \mathrm{H}), 1.33-1.21(1 \mathrm{H})$, and 0.89-0.68 $(4 \mathrm{H}),{ }^{13} \mathrm{C}-\mathrm{NMR}\left(\mathrm{CDCl}_{3}\right.$, $75 \mathrm{MHz}) \delta 152.00\left(\mathrm{C}_{\mathrm{q}}\right), 150.69(\mathrm{CH}), 150.35\left(\mathrm{C}_{\mathrm{q}}\right), 147.34\left(\mathrm{C}_{\mathrm{q}}\right) ; 135.10(\mathrm{CH}), 132.16(\mathrm{CH})$, 125.65 $\left(\mathrm{C}_{\mathrm{q}}\right), 121.59(\mathrm{CH}), 119.93(\mathrm{CH}), 119.42(\mathrm{CH}), 112.46(\mathrm{CH}), 69.27(\mathrm{CH}), 63.47\left(\mathrm{CH}_{2}\right)$, $60.59\left(\mathrm{CH}_{2}\right), 60.33(\mathrm{CH}), 57.67\left(\mathrm{CH}_{2}\right), 57.43\left(\mathrm{CH}_{2}\right), 54.13\left(\mathrm{CH}_{2}\right), 40.22\left(\mathrm{CH}_{2}\right), 36.83(\mathrm{CH})$, $33.10(\mathrm{CH}), 27.40(\mathrm{CH}), 27.00(\mathrm{CH}), 26.89\left(\mathrm{CH}_{2}\right), 25.16\left(\mathrm{CH}_{2}\right), 24.79\left(\mathrm{CH}_{2}\right), 20.99(\mathrm{CH}), 20.10$ $\left(\mathrm{CH}_{2}\right)$, and $11.83\left(\mathrm{CH}_{3}\right)$. Melting point: the substance decomposed at $\mathrm{T}=121{ }^{\circ} \mathrm{C}$; TLC (TBME: $\mathrm{MeOH}$ : ammonia 25\%/100:10:1): rf-value = 0.14; IR: see spectrum in the supporting information; HR-MS (ESI, MeOH) calculated for $\mathrm{C}_{29} \mathrm{H}_{38} \mathrm{~N}_{6} \mathrm{O}_{2}+\mathrm{H}^{+}$: $503.31290 \mathrm{Da}$, found 503.31306 Da.

\subsubsection{X-ray Structure Determination}

The single crystals were mounted on a Hampton loop using perfluoroether oil and placed in the cold nitrogen gas stream on the diffractometer [29]. The data were collected on a Rigaku Oxford Diffraction Synergy-S using mirror-focused $\mathrm{CuK}_{\alpha}$ radiation. The reflections were indexed, integrated, and appropriate absorption corrections were applied as implemented in the CrysAlisPro software package [30]. The structures were solved employing the program SHELXT and refined an isotropically for all non-hydrogen atoms by full-matrix least squares on all F2 using SHELXL software [31,32]. Carbon bound hydrogen atoms were refined employing a riding model; methyl groups were treated as rigid bodies and were allowed to rotate about the E-CH3 bond. Nitrogen and oxygen bound hydrogen atoms were located in the difference Fourier map and were refined freely for $\mathbf{1 0}$ and 11, whilst for $\mathbf{2 8}$ geometrical restraints and constraints for the displacement parameters were employed. For 28, the structure contains a partly occupied $\mathrm{HCl}$ and two 
partly occupied units of water. The absolute structure parameter suggests numerically a very small contribution of an inversion twined component. As the only heavy atom (Cl1) is only partly occupied, a twin refinement was considered not reliable and was not conducted. During refinement and analysis of the crystallographic data, the programs OLEX2 and Diamond were used $[33,34]$. Unless noted otherwise, the shown ellipsoids represent the $50 \%$ probability level and hydrogen atoms are displayed with an arbitrary radius.

Supplementary Materials: The following are available online. Hardcopy from 1H-NMR and 13CNmr experiments; IR spectrograms and Table S1: Crystallographic data collection parameter of 10, 11 and 28.

Author Contributions: Conceptualization, I.N., C.V.M. and M.H.F.; methodology, I.N. and C.V.M.; validation, I.N. and C.V.M.; formal analysis, M.H.F.; investigation, C.V.M., M.H.F., and C.K.; resources, C.V.M. and M.H.F.; data curation, C.V.M. and M.H.F.; writing-original draft preparation, M.H.F. and C.V.M.; writing-review and editing, I.N. and C.V.M.; supervision, I.N.; project administration, I.N.; funding acquisition, I.N. All authors have read and agreed to the published version of the manuscript.

Funding: This research received no external funding.

Institutional Review Board Statement: Not applicable.

Informed Consent Statement: Not applicable.

Data Availability Statement: The data presented in this study are available on request from the corresponding author. Crystallographic data have been deposited with the Cambridge Crystallographic Data Centre as supplementary publications no. CCDC-2080994(10), -2080992(11), -2080993(28). Copies of the data can be obtained free of charge from www.ccdc.cam.ac.uk/data_request/cif (accessed on 30 April 2021).

Acknowledgments: We want to thank Buchler $\mathrm{GmbH}$, Braunschweig, Germany for making this work possible through a generous donation of the starting Amines $\mathbf{1}$ to $\mathbf{6}$.

Conflicts of Interest: The authors declare no conflict of interest.

Sample Availability: Samples of the compounds 1 to 6 and 8 to 13 are available from the authors.

\section{References}

1. Hashimoto, Y. Biochemical studies on quinoline derivatives. IV. Properties of quinoline dehydrogenase. Nihon Univ. J. Med. 1960, 2, 229-245.

2. Marcelli, T.; van Maarseveen, J.H.; Hiemstra, H. Cupreines and Cupreidines: An Emerging Class of Bifunctional Cinchona Organocatalysts. Angew. Chem. Int. Ed. 2006, 45, 7496-7504. [CrossRef] [PubMed]

3. Bryant, L.A.; Fanelli, R.; Cobb, A.J.A. Cupreines and cupreidines: An established class of bifunctional cinchona organocatalysts. Beilstein J. Org. Chem. 2016, 12, 429-443. [CrossRef] [PubMed]

4. Nakatsuji, M.; Fujita, M.; Okamoto, Y.; Sugimura, T. Kinetic analysis of the asymmetric hydrogenation of (E)-2,3diphenylpropenoic acid over cinchonidine derivative-modified Pd/C: Quinoline ring modification. Catal. Sci. Technol. 2020, 10, 6573-6582. [CrossRef]

5. Neda, I.; Kaukorat, T.; Hrib, C.G. New hydrogenated and didehydrogenated 1,2-diamines of quincorine and quincoridine. Tetrahedron Asymmetry 2002, 13, 1327-1330. [CrossRef]

6. Neda, I.; Kaukorat, T.; Fischer, A.K. Unusual stabilization of 1,2-diamino derivatives of quincorine and quincoridine by carbon dioxide: Persistent crystalline prim-ammonium-carbamate salts and their reactivity towards isatoic acid anhydride. Eur. J. Org. Chem. 2003, 3784-3790. [CrossRef]

7. Maftei, C.V.; Fodor, E.; Jones, P.G.; Franz, M.H.; Davidescu, C.M.; Neda, I. Asymmetric calixarene derivatives as potential hosts in chiral recognition processes. Pure Appl. Chem. 2015, 87, 415-439. [CrossRef]

8. Neda, I.; Fodor, E.; Maftei, C.V.; Mihorianu, M.; Ambrosi, H.D.; Franz, M.H. New members of the cinchona alkaloid family: 9-aminoquincorine-10-aldehyde and 9-aminoquincoridine-10-aldehyde. Eur. J. Org. Chem. 2013, 7876-7880. [CrossRef]

9. Maftei, E.; Maftei, C.V.; Franz, M.H.; Kelter, G.; Fiebig, H.H.; Tamm, M.; Neda, I. New members of the Cinchona alkaloid family: Synthesis, characterisation and antitumor evaluation of novel Gold(I) complexes. Rev. Roum. Chim. 2016, 61, $251-260$.

10. Franz, M.H.; Röper, S.; Wartchow, R.; Hoffmann, H.M.R. The First and Second Cinchona Rearrangement. Two Fundamental Transformations of Alkaloid Chemistry. J. Org. Chem. 2004, 69, 2983-2991. [CrossRef] 
11. Maftei, C.V.; Fodor, E.; Jones, P.G.; Freytag, M.; Franz, M.H.; Kelter, G.; Fiebig, H.H.; Tamm, M.; Neda, I. N-heterocyclic carbenes (NHC) with 1,2,4-oxadiazole-substituents related to natural products: Synthesis, structure and potential antitumor activity of some corresponding gold(I) and silver(I) complexes. Eur. J. Med. Chem. 2015, 101, 431-441. [CrossRef] [PubMed]

12. Filimon, S.A.; Hrib, C.G.; Randoll, S.; Neda, I.; Jones, P.G.; Tamm, M. Quinine-Derived Imidazolidin-2-imine Ligands: Synthesis, Coordination Chemistry, and Application in Catalytic Transfer Hydrogenation. Z. anorg. allg. Chem. 2010, 636, 691-699. [CrossRef]

13. Bota, S.; Neda, I.; Dumitrescu, L.S. Synthesis and HPLC Separation of New Quinuclidine Derivatives. Rev. Roum. Chim. 2012, 57, 281-286.

14. Franz, M.H.; Birzoi, R.; Maftei, C.V.; Maftei, E.; Kelter, G.; Fiebig, H.H.; Neda, I. Studies on the constituents of Helleborus purpurascens: Analysis and biological activity of the aqueous and organic extracts. Amino Acids 2018, 50, 163-188. [CrossRef] [PubMed]

15. Franz, M.H.; Iorga, M.; Maftei, C.V.; Maftei, E.; Neda, I. Studies on the constituents of Helleborus purpurascens: Use of derivatives from calix [6]arene, homooxacalix[3]arene and homoazacalix[3]arene as extractant agents for amino acids from the aqueous extract. Amino Acids 2019, 52, 55-72. [CrossRef]

16. Maftei, E.; Maftei, C.V.; Freytag, M.; Franz, M.H.; Bannenberg, T.; Neda, I. Clarification of stereochemistry aspects for N-hydroxy5-norbornene-2,3-dicarboximide derivatives and elucidation of them by experimental and theoretical investigations, including the synthesis of N, N'-bis-(5-exo-norbornene-2,3-dicarboxyimidyl) carbonate. Rev. Roum. Chim. 2018, 63, 245-255.

17. Röper, S.; Franz, M.H.; Wartchow, R.; Hoffmann, H.M.R. Fused triazoles via tandem reactions of activated Cinchona alkaloids with azide ion. Second Cinchona rearrangement exemplified. Org. Lett. 2003, 5, 2773-2776. [CrossRef] [PubMed]

18. Zhao, Y.; van Nguyen, H.; Male, L.; Craven, P.; Buckley, B.R.; Fossey, J.S. Phosphino-Triazole Ligands for Palladium-Catalyzed Cross-Coupling. Organometallics 2018, 37, 4224-4241. [CrossRef]

19. Singh, M.S.; Chowdhury, S.; Koley, S. Advances of azide-alkyne cycloaddition-click chemistry over the recent decade. Tetrahedron 2016, 72, 5257-5283. [CrossRef]

20. Martzel, T.; Annibaletto, J.; Levacher, V.; Brière, J.F.; Oudeyera, S. C5-Disubstituted Meldrum's Acid Derivatives as Platform for the Organocatalytic Synthesis of C3-Alkylated Dihydrocoumarins. Adv. Synth. Catal. 2019, 361, 995-1000. [CrossRef]

21. Zhang, Y.; Wang, M.; Li, J.; Di, Q.; Tian, Z.; Chen, B.; Zhang, H.; Tanimoto, H.; Morimoto, T.; Kakiuchi, K. Acid Promoted Metal Free Synthesis of Trizole-Fused Heterocycles via Intramolecular [3 + 2] Cycloaddition. Heterocycles 2018, 96, 943-953. [CrossRef]

22. Gayen, F.R.; Ali, A.A.; Bora, D.; Roy, S.; Saha, S.; Saikia, L.; Goswameea, R.L.; Saha, B. A ferrocene functionalized Schiff base containing $\mathrm{Cu}(\mathrm{II})$ complex: Synthesis, characterization and parts-per-million level catalysis for azide alkyne cycloaddition. Dalton Trans. 2020, 49, 6578-6586. [CrossRef]

23. Cwiklicki, A.; Rehse, K. Antiaggregating and Antithrombotic Activities of new 1,2,3-Triazolecarboxamides. Arch. Pharm. Pharm. Med. Chem. 2004, 337, 156-163. [CrossRef] [PubMed]

24. Liu, M.; Hou, Y.; Yin, W.; Zhou, S.; Qian, P.; Guo, Z.; Xu, L.; Zhao, Y. Discovery of a novel 6,7-disubstituted-4-(2fluorophenoxy)quinolines bearing 1,2,3-triazole-4-carboxamide moiety as potent c-Met kinase inhibitors. Eur. J. Med. Chem. 2016, 119, 96-108. [CrossRef]

25. Pirali, T.; Tron, G.C.; Zhu, J. One-Pot Synthesis of Macrocycles by a Tandem Three-Component Reaction and Intramolecular [3 + 2] Cycloaddition. Org. Lett. 2006, 8, 4145-4148. [CrossRef] [PubMed]

26. Zakharova, E.A.; Shmatova, O.I.; Nenajdenko, V.G. Acetylene-azide click macrocyclization of peptides. Russ. Chem. Rev. 2018, 87, 619-635. [CrossRef]

27. Bahulayan, D.; Arun, S. An easy two step synthesis of macrocyclic peptidotriazoles via a four-component reaction and copper catalyzed intramolecular azide-alkyne [3 + 2] click cycloaddition. Tetrahedron Lett. 2012, 53, 2850-2855. [CrossRef]

28. Jasinski, R.; Dresler, E. On the Question of Zwitterionic Intermediates in the [3 + 2] Cycloaddition Reactions: A Critical Review. Organics 2020, 1, 49-69. [CrossRef]

29. Stalke, D. Cryo crystal structure determination and application to intermediates. Chem. Soc. Rev. 1998, 27, 171-178. [CrossRef]

30. Rigaku Oxford Diffraction, Versions 1.171.41.96a-105a; CrysAlisPro; OlexSys: Durham, UK, 2021.

31. Sheldrick, G.M. SHELXT-Integrated space-group and crystal-structure determination. Acta Cryst. 2015, A71, 3-8. [CrossRef]

32. Sheldrick, G.M. Crystal structure refinement with SHELXL. Acta Cryst. 2015, C71, 3.

33. Dolomanov, O.V.; Bourhis, L.J.; Gildea, R.J.; Howard, J.A.K.; Puschmann, H. OLEX2: A Complete Structure Solution, Refinement and Analysis Program. J. Appl. Cryst. 2009, 42, 339-341. [CrossRef]

34. Putz, H.; Brandenburg, K. Diamond-Crystal and Molecular Structure Visualization; Crystal Impact-GbR: Bonn, Germany, 2020. 